\title{
Die Banquette des Val du Bourget (franz. Nordalpen): Gelöste und ungelöste Probleme der jungpleistozänen Klima- und Reliefentwicklung
}

\author{
Christian Hannss \& Peter Peschke *) \\ Upper Pleistocene, banquette, climate, morphology, stratigraphy, \\ France, northern Alps
}

\begin{abstract}
Kurzfassung: Im Val du Bourget der französischen Alpen sind Banquette entwickelt, die ihre Entstehung im wesentlichen dem Spätpleistozän verdanken. Ihr litho-, bio- und chronostratigraphischer Aufbau wird vor dem Hintergrund dreier neuer Pollenanalysen und eines neuen Aufschlusses untersucht. Die Ergebnisse werden mit anderen europäischen Typlokalitäten des Spätpleistozäns verglichen.
\end{abstract}

[Les banquettes du Val du Bourget, Alpes françaises du Nord: Problèmes résolus et non résolus de l'évolution climatique et géomorphologique au Pleistocène supérieur]

Résumé: On observe dans le Val du Bourget des Alpes françaises du Nord des banquettes. Elles se sont formées essentiellement au cours du Pleistocène supérieur. Leur structures litho-, bio- et chronostratigraphiques sont étudiées et mis en rapport avec trois nouveaux diagrammes polliniques et une nouvelle coupe. Les résultats obtenus sont comparés à d'autres sites classiques du Pleistocène supérieur en Europe.

[The banquettes of the Val du Bourget, northern French Alps: Problems resolved and not resolved of the climatic and geomorphological development during the upper Pleistocene]

Abstract: In the Val du Bourget of the northern French Alps "banquettes" are developped. Their litho-, bio- and chronostratigraphic structure are discussed in respect to three new pollen records and a new cut. The results are compaired with other famous sites of the upper Pleistocene in Europe.

*) Anschriften der Verfasser: CH. Hannss, Geographisches Institut der Universität Tübingen, Hölderlinstr. 12, D - 7400 Tübingen 1. P. PESCHKE, Universität Hohenheim, Botanik 210, Postfach 700 562, D - 7000 Stuttgart 70.

\section{Lage und Bau des Untersuchungsraumes}

Das Val du Bourget schiebt sich zwischen die Cluse von Chambéry im Süden und den Lac du Bourget im Norden. Die Cluse von Chambéry trennt die Chartreuse im SSW von den Bauges im NNE. Der Lac du Bourget wie das Val du Bourget werden nicht mehr von den westlichen französischen Kalkalpen, sondern von niedrigeren, bereits zum Jurasystem gehörenden Höhenzügen aus jurassischen bis miozänen, meist karbonatischen Sedimentgesteinen gebildet (DEBELMAS 1970: 10).

\section{Die geomorphologischen Grundzüge}

Neben der Felsenumrahmung charakterisieren noch Banquette und die holozäne Schwemmlandebene der Leysse das Reliefbild des Val du Bourget (Abb. 1). Die Banquette, die einen Kern aus Anstehendem aufweisen, erheben sich terrassenähnlich über die postglaziale Leysse-Schwemmlandebene, ähnlich wie das „Mittelgebirge" der Inntal-Terrassen über die holozäne Aue des Inns. Auch genetisch sind sich diese jungpleistozänen Akkumulationsformen ähnlich. Beide werden nämlich im Liegenden von lakustren Akkumulationen aufgebaut, die fluviatile Sedimente überlagern. In diese Ablagerungen haben sich würmzeitlich noch Talgletscher eingetieft, die stellenweise mächtige Moränendecken auf den Schottern hinterlassen haben.

Physiognomisch weisen die oberflächlich leicht gewellten Banquette des Val du Bourget eine gewisse Ähnlichkeit mit niedrigen Trogschultern auf. Randlich sind ebenso wie in die westlich der Leysse gelegenen Banquette von Tremblay wie auch in die sich 


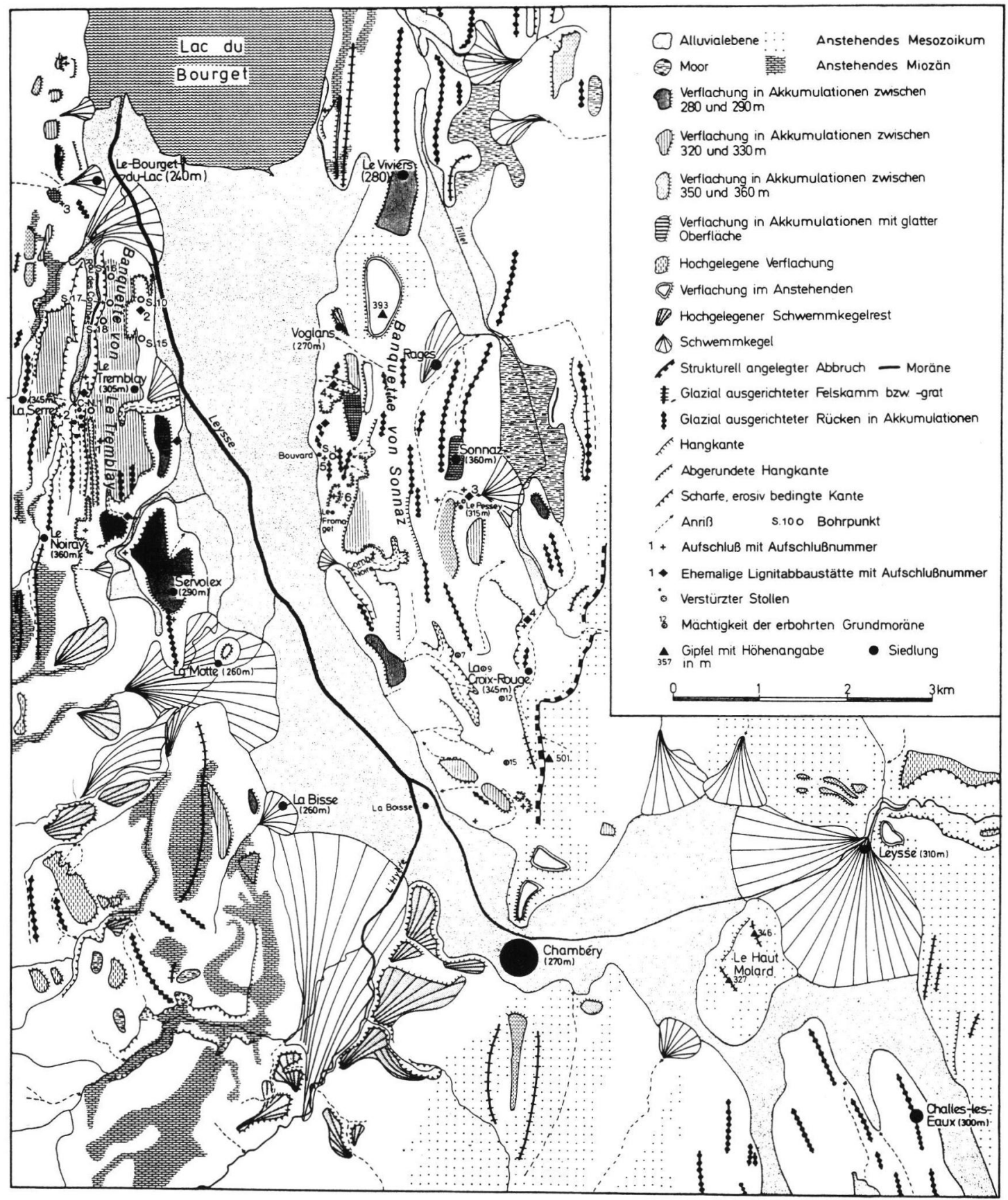

Abb. 1: Die geomorphologischen Grundzüge des Val du Bourget.

Die Aufschlußnummern beinhalten:

1 = Kiesgrube östlich Le Fort

2 = Kiesgrube auf der linken Seite des Rau des Combes

3 = Unterhalb Les Ciseaux
4 = Kiesgrube Voglans

5 = Voglans-Bouvard

Der Aufschluß ,Pont de la Charrière’ liegt zwischen der Aufschlußnummer 2 und der ehemaligen Lignitabbaustätte Nr. 1.

Die Nummern der ehemaligen Lignitabbaustätten beinhalten:

1 = Alte Lignitmine Rau des Combes

2 = Alte Lignitmine in der Kiesgrube Aéroport du Bourget
3 = Alte Lignitmine Pessey-Sonnaz

4 = Alte Lignitmine La Croix-Rouge 
östlich dieses kleinen Flusses erhebenden Banquette von Sonnaz stellenweise noch terrassenförmige Flachformen eingesenkt. Sie ordnen sich in verschiedenen Niveaus, und zwar in $360 \mathrm{~m}-350 \mathrm{~m}, 330 \mathrm{~m}-320 \mathrm{~m}$ und in $290 \mathrm{~m}-280 \mathrm{~m} \mathrm{~N}$. N. an. Sie dürften ihre Entstehung in erster Linie der Lateralerosion von Schmelzwässern des etappenweise zurückweichenden würmzeitlichen Isèregletschers verdanken. Aus Schwermineralanalysen hat sich nämlich ergeben, daß wenigstens im Würm die Cluse von Chambéry und das Val du Bourget in nördlicher Richtung von einem Teilast des Isère-Gletschers durchströmt wurden (HANNSS 1982: 104).

\section{Der lithostratigraphische Aufbau der Banquette im Val du Bourget}

\subsection{Die allgemeinen Grundcharakteristika}

Eine immer wiederkehrende Grundabfolge der jungpleistozänen Lockersedimente im Bereich der Banquette des Val du Bourget besteht von unten nach oben aus moränenunterlagerten tonigen bis sandigen Feinsedimenten, die von bis zu $4 \mathrm{~m}$ mächtigen Schieferkohlen durchsetzt bzw. überlagert werden. Darüber wurden meist diskordant und nach oben in der Regel gröber und sandärmer werdende Schotter von bis zu $20 \mathrm{~cm}$ Durchmesser akkumuliert (Abb. 2). Letztere werden häufig noch von einer tonigen, kalkreichen Grundmoräne überlagert.
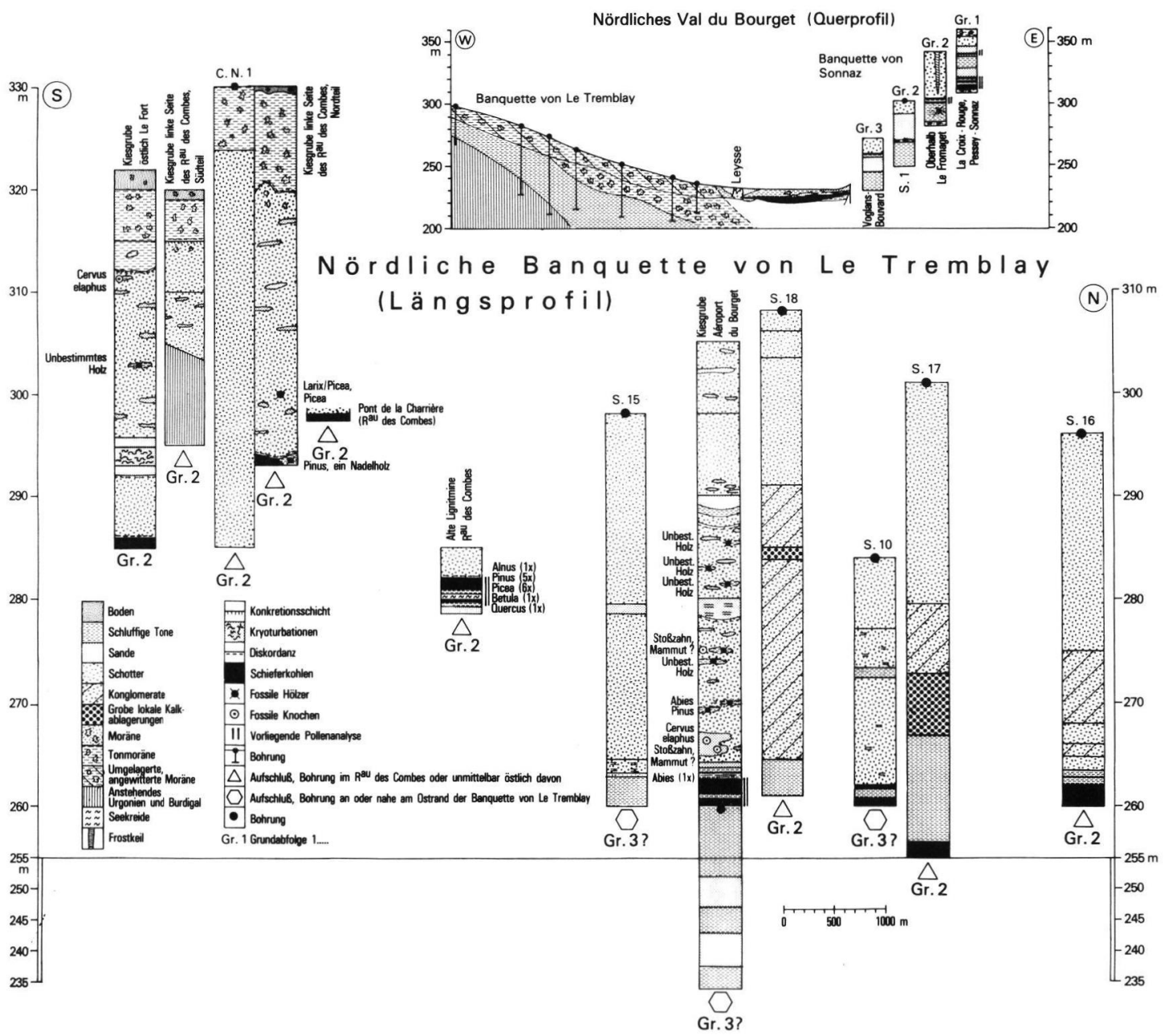

Abb. 2: Lithostratigraphische Grundstrukturen der Banquette im Val du Bourget.

Der Westteil des Querprofils wurde nach Nicoud 1979: 869 gezeichnet.

Die Lage der Bohrprofile und der Aufschlüsse ist Abb. 1 zu entnehmen. 
Tabelle 1: Schematische Darstellung der Grundabfolgen 1 bis 3 aus den spätpleistozänen Banquetteablagerungen des Val du Bourget. Nach Gremmen 1982; HanNss 1982; Renault-MisKovsky 1986, Woillard 1978 und Wolltard, Mook 1982.

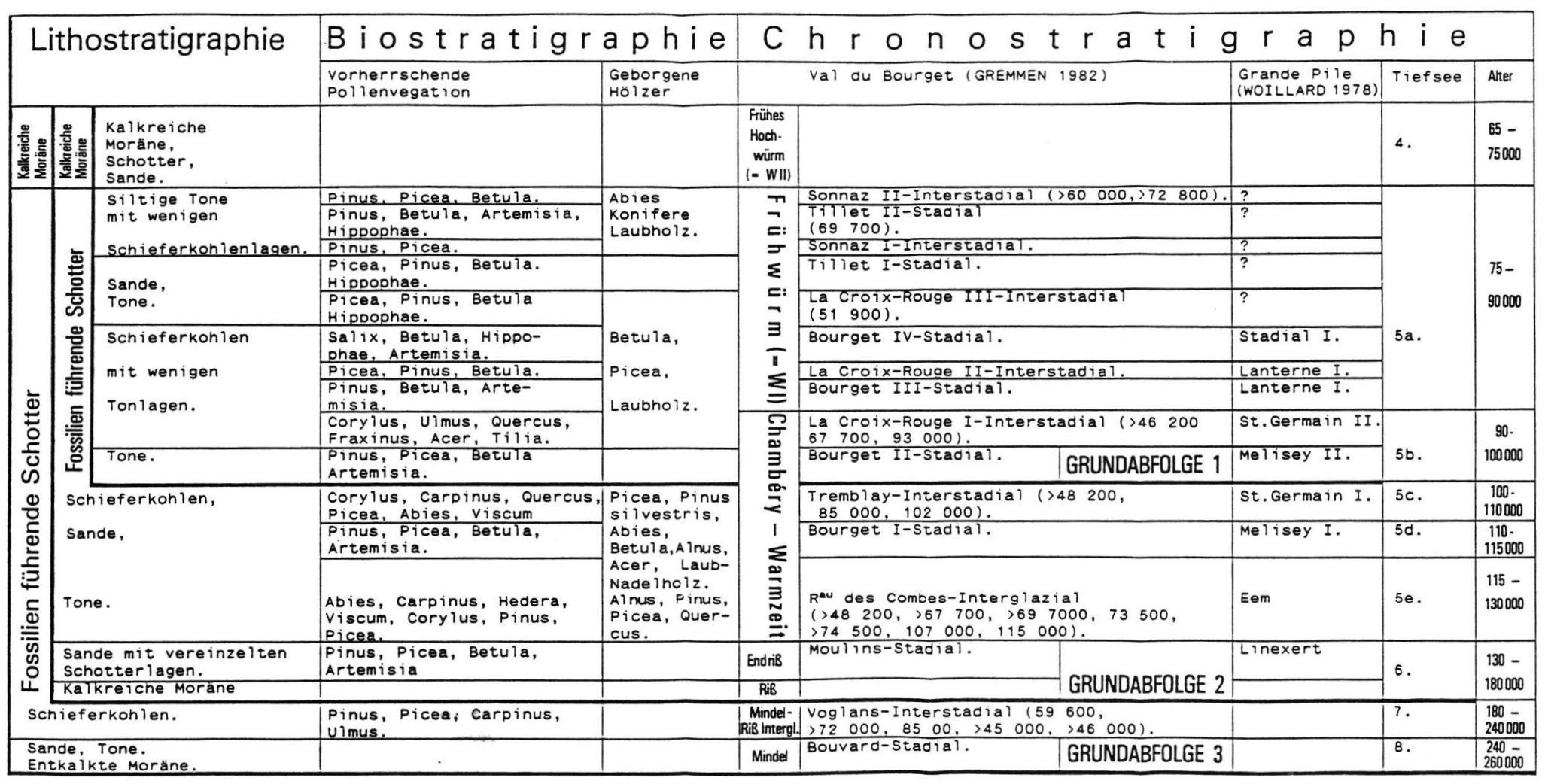


In Anlehnung an die morphologisch erfaßte glaziale Serie von A. PENCK (SCHAEFER 1981: 273) könnte man diese Akkumulationsabfolge als ,pleistozänen Sedimentationszyklus" bezeichnen. Er tritt in dieser Form nicht nur im Val du Bourget, sondern prinzipiell auch in der Combe de Savoie und im Becken von Grenoble auf (HANNSS, 1982: Tafel II, Abb. 6). Er spiegelt eine ältere Vergletscherung, die Füllung eines Eisrückzugssees, mitunter Moorbildungen, die Geröllakkumulation in der Form mehr oder weniger prägnant ausgebildeter Vorstoßschotter im Vorfeld einer neuen Vereisungsphase wider.

\subsection{Die Grundabfolge 1}

Die Grundabfolge 1 ist im Gegensatz zu den anderen beiden nur im Bereich der Banquette von Sonnaz ausgebildet, und zwar zwischen $307 \mathrm{~m}$ und $360 \mathrm{~m} \mathrm{~N}$. N. Die Sequenz besteht im Liegenden aus fossilführenden Tonen von mindestens $5 \mathrm{~m}$ Mächtigkeit, die im obersten Teil Fossilien führen. Darüber sind zwischen $312 \mathrm{~m}$ und $322 \mathrm{~m}$ in nach oben zunehmender Zahl und Mächtigkeit mehrere Schieferkohlenhorizonte entwickelt. Sie werden in der Regel von stärker tonigschluffigen Feinsedimenten, in $316 \mathrm{~m}$ jedoch auch noch von einer knapp einen Meter mächtigen fluviatilen Schotterlage voneinander getrennt. Darüber wurden rund $20 \mathrm{~m}$ mächtige, abgesehen von vereinzelten Pollen, fossilleere Tone, Schluffe und Sande akkumuliert. Sie werden in $341 \mathrm{~m}$ noch einmal von einer dreifach gegliederten Schieferkohlen-Sequenz abgelöst.

In Pessey-Sonnaz treten über den mit $341 \mathrm{~m}$ bisher höchsten im Val du Bourget gefundenen Schieferkohlen noch knapp $10 \mathrm{~m}$ mächtige Feinablagerungen in Form geschichteter Sande auf. Darüber folgen Schotter und eine kalkreiche würmzeitliche Moräne. Die einzelnen Schichtglieder der Grundabfolge 1 lagern zumindest in den unteren Partien transgressiv einer entkalkten, mit Sicherheit präwürmzeitlichen, wahrscheinlich bereits aus der Mindeleiszeit stammenden Moräne auf (Abb. 2; Tabelle 1).

\subsection{Die Grundabfolge 2}

Im Gegensatz zur Grundabfolge 1 findet man die verschiedenen Schichtglieder der Grundabfolge 2 sowohl im Bereich der Banquette von Le Tremblay wie der von Sonnaz, und zwar zwischen $340 \mathrm{~m}$ und $258 \mathrm{~m}$ N. N. Der Hauptschieferkohlenhorizont der Grundabfolge 2 tritt in einer weiten Höhenstreuung zwischen $268 \mathrm{~m}$ und $305 \mathrm{~m}$ auf. Dabei konstatiert man, daß die Lignite an den gebirgsseitigen, äußeren Rändern, dort wo sie in einem geringeren vertikalen Abstand zu den miozänen Sandsteinen und Konglo- meraten des anstehenden Burdigal und Torton auftreten, höhere Positionen einnehmen als weiter zur Talmitte hin, wo die leichter komprimierbaren liegenden Pleistozänablagerungen in größerer Mächtigkeit ihren Untergrund bilden. An zwei Stellen wurde zudem festgestellt, daß die Schieferkohlen der stratigraphischen Grundabfolge 2 zusätzlich noch um wenige Grade in der Längsachse des Val du Bourget, und zwar ebenso nach Norden wie auch nach Süden, einfallen.

Die Lignite der Grundabfolge 2 werden wie die der Grundabfolge 1 von sandig-tonigen Feinakkumulationen unterlagert, denen nur im oberen Teil an ganz wenigen Stellen Schotterlagen zwischengeschaltet sind. In der Kiesgrube von Voglans wurden 1991 unter diesen Feinablagerungen, und zwar zwischen $270 \mathrm{~m}$ und mindestens $266 \mathrm{~m} \mathrm{~N}$. N., kalkreiche Moränenformationen kartiert. Sie sind höchstwahrscheinlich mit den Glazialablagerungen zu korrelieren, die in Voglans über den unteren, den $250 \mathrm{~m}$-Schieferkohlen anstehen (HANNSS 1982: 68, Schichtglied 18).

Die Schieferkohlen der Grundabfolge 2 weisen auf kürzesten Horizontaldistanzen große fazielle und Mächtigkeitsunterschiede auf. Sie keilen zudem nachweislich in bestimmten Bereichen auch aus.

Dort, wo die Lignite der Grundabfolge 2 ausgebildet sind, werden sie in der Regel längs einer ausgeprägten Erosionsdiskordanz von Schottern überdeckt. Bisweilen wurden die Schieferkohlen durch fluviatile Erosion bis auf wenige Reste fast ganz abgetragen. Stellenweise hat sich die Sedimentation minerogenen Feinmaterials jedoch auch nach der Ausbildung der Schieferkohlenformationen noch eine Zeit lang fortgesetzt.

In der Kiesgrube von Voglans konnte an einer Stelle beobachtet werden, daß die Schieferkohlenflöze der Grundabfolge 2 wohl verwürgt, aber ohne jeden deutlichen Schichten-Hiatus in die hangenden Schotterformationen übergehen (Abb. 3). An einer anderen Stelle zeigen sie hier dagegen nur schwer deutbare Unter- und Einlagerungen von Schottern. Möglicherweise hängen diese sehr eigenartigen lithostratigraphischen Strukturen mit glazio-tektonischen Deformationen zusammen, zu denen es im Zusammenhang mit der würmzeitlichen Eisüberfahrung dieser wahrscheinlich aus dem letzten Interglazial stammenden Schieferkohlenformation gekommen ist.

Daß auf die Ablagerung der Schieferkohlen der Grundabfolge 2 stadialzeitliche Klimabedingungen folgten, ergibt sich aus eindeutigen Kryoturbationsstrukturen, die im Bereich der Banquette von Le Tremblay in einer $2 \mathrm{~m}$ mächtigen, in $292 \mathrm{~m}$ Höhe N.N. ansetzenden Sandlinse in der Kiesgrube östlich 


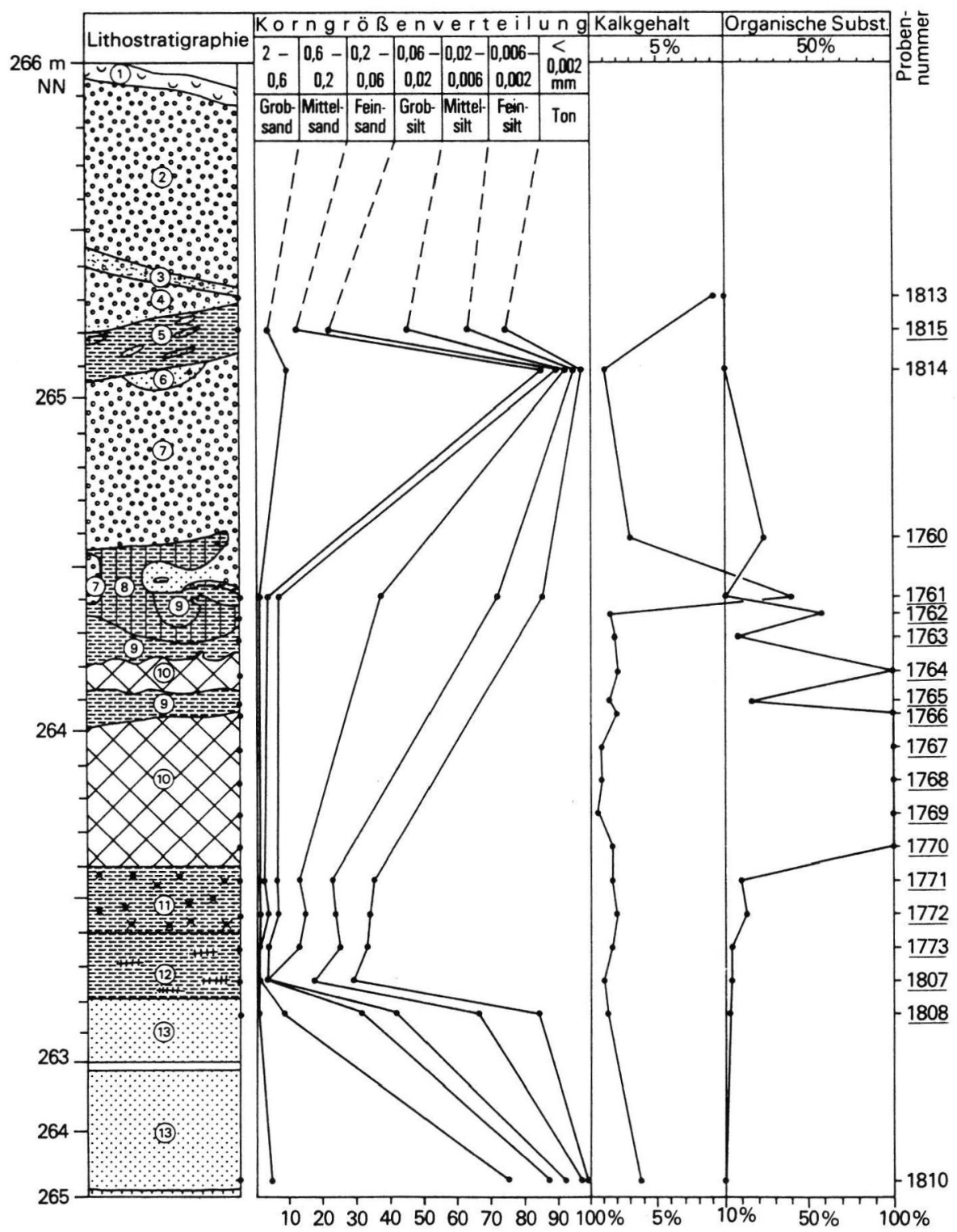

Abb. 3: Lithostratigraphie und Sedimentologie der späteemzeitlichen $266 \mathrm{~m}$-Lignite in der Kiesgrube von Voglans (Grundabfolge 2).

$1=$ Rezenter Boden

$2=$ Gut gerundete Schotter

3 = Stärker sandiges Lehmband mit vereinzelten Geröllen

4 = Nach oben gröber werdende Gerölle mit bis zu $10 \mathrm{~cm}$ Durchmesser, die nach unten über Sande in Lehme übergehen

$5=$ Fette graue bis lilafarbene Tone, die mit kleinen Sandlinsen und Lignittonen durchsetzt sind

6 = Mittelsande

7 = Uneingeregelte Schotter mit durchschnittlich $5 \mathrm{~cm}$ Durchmesser, die im Liegenden teilweise in verwürgte Grobsande übergehen

8 = Verwürgte, rötlich-braune Lignittone

9 = Unterschiedlich intensiv verwürgte rötlich-graue Tone

$10=$ Schieferkohlenflöze, zum Teil leicht verwürgt

11 = Plastische, mit vielen pflanzlichen Makroresten durchsetzte hellgrau-bläuliche Tone

12 = Fette, grau-blaue Tone, die lagenweise rötlich oxidiert sind und keine Pollen mehr enthalten

13 = Grünlich-graue, nach oben leicht tonige Sande, die in $265,25 \mathrm{~m} \mathrm{~N}$. N. unter einer $1 \mathrm{~cm}$ mächtigen Eisenkonkretionsschicht in gröbere braune Sande übergehen. Sie erwiesen sich wie die Schicht 12 als pollenleer 
Le Fort, nur $5 \mathrm{~m}$ über den liegenden Schieferkohlen beobachtet wurde (Abb. 2).

Auf wesentlich kühlere Sedimentationsbedingungen nach der Herausbildung der Hauptschieferkohlenflöze der Grundabfolge 2 verweisen überdies aus lokalem Kalkmaterial bestehende Murschuttablagerungen, die im Zusammenhang mit Deltastrukturen zwischen $285 \mathrm{~m}$ und $290 \mathrm{~m} \mathrm{~N}$. N. im Norden der Banquette von Le Tremblay beobachtet wurden (Abb. 2; HANNSS 1982: 95, 96).

Im Zusammenhang mit der hochwürmzeitlichen Eisüberfahrung sind schmale, aber sehr lange, mit sandig-tonigem Feinmaterial verfüllte Eiskeile $\mathrm{zu}$ sehen, die in den Schottern der Grundabfolge 2 oberhalb von Le Fromaget kartiert wurden (Abb. 4).

Litho-, und wie auch noch gezeigt wird biostratigraphisch ist an der Existenz der Grundabfolge 1 und 2 im Val du Bourget nicht zu zweifeln. Der direkte lithostratigraphische Konnex zwischen beiden konnte jedoch bisher noch nicht beobachtet werden. Da die höchsten Schieferkohlenflöze der Grundabfolge 2 oberhalb von Le Fromaget mit $305 \mathrm{~m}$ N. N. jedoch nur $7 \mathrm{~m}$ unter dem Hauptlignitniveau der Grundabfolge 1 von Pessey-Sonnaz anstehen, ist es durchaus möglich, daß die Grundabfolge 1 das mehr oder weniger unmittelbar Hangende der Grundabfolge 2 darstellt

\subsection{Die stratigraphische Grundabfolge 3}

Obwohl biostratigraphisch der letzte Beweis noch fehlt, kann auf Grund jüngster lithostratigraphischer Beobachtungen in der Kiesgrube von Voglans die Existenz der Grundabfolge 3 heute als nahezu gesichert angesehen werden.

Für eine selbständige Grundabfolge 3 sprechen einmal die auf beiden Seiten des. Val du Bourget in Höhen zwischen $240 \mathrm{~m}$ und $260 \mathrm{~m} \mathrm{~N}$. N. entwickelten Schieferkohlen. Sie liegen damit wesentlich tiefer als die zwischen $268 \mathrm{~m}$ und $305 \mathrm{~m} \mathrm{~N}$. N. beobachteten Hauptlignitzflöze der stratigraphischen Grundabfolge 2 (HANNSS 1982: 6).

Gestützt wird die Hypothese von einer dritten untersten Grundabfolge zum anderen dadurch, daß am Unterlauf des $\mathrm{R}^{\mathrm{au}}$ des Combes in der Bohrung S. 17 in $255 \mathrm{~m}$ Höhe Schieferkohlen angefahren wurden, die noch von $10 \mathrm{~m}$ mächtigen, tonigen Feinsedimenten überlagert werden, bevor sie in die hangenden Schotter übergehen (Abb. 2).

Für die Existenz einer Grundabfolge 3 spricht zudem die Tatsache, daß auf der Ost-Seite der nördlichen Banquette von La Tremblay in den in der Kiesgrube Aéroport du Bourget anstehenden Schottern in Höhen um $280 \mathrm{~m}$ mächtige Sand- und Tonlinsen auftreten, in denen etwas weiter westlich, am mittleren $\mathrm{R}^{\text {au }}$ des Combes, das Hauptlignitflöz der Grundabfolge 2 bergmännisch ausgebeutet wurde (Abb. 2).

$\mathrm{Daß}$ in den Banquetten des Val du Bourget nicht nur zwei, sondern drei Hauptlignitflöze entwickelt sind, ergibt sich vor allem aber aus der Moränenlage, die $199110 \mathrm{~m}$ unter den $280 \mathrm{~m}$-Schieferkohlen der Grundabfolge 2 in der Kiesgrube von Voglans beobachtet worden ist. Im Unterschied zu den völlig entkalkten Moränenformationen, welche das Liegende der Feinablagerungen unter den $250 \mathrm{~m}$-Ligniten der Grundabfolge 3 bilden, sind die Glazialakkumulationen, welche die Basis der Grundabfolge 2 darstellen, jedoch ähnlich kalkreich wie die hangende Würmmoräne der Grundabfolge 1.

Gegen die Existenz einer dritten Grundabfolge spricht eigentlich nur der Umstand, daß alle Lignitflöze der Banquette im Val du Bourget generell zur Talmitte hin einfallen, und die Schieferkohlen der Grundabfolge 3 bisher nur an den Innenseiten der Banquette des Val du Bourget beobachtet werden konnten (HANNSS, 1982: Abb. 31, 32, Tafel III).

Nicoud (1979: 859-860) zufolge dürfte die Grundabfolge 3 nicht existieren. Er geht davon aus, daß alle Schieferkohlenflöze des Val du Bourget ursprünglich in gleicher Höhe entstanden sind und erst nachträglich durch Tektonik, insbesondere Staffelbrüche, in ihre heutige Lage geraten sind. Daß diese Hypothese jedoch die sehr unterschiedlichen Höhenlagen der Schieferkohlenflöze allein nicht erklären kann, ergibt sich, abgesehen von den pollenanalytischen Ergebnissen, auch daraus, daß auf der Halbinsel Grésine nördlich von Aix-Les-Bains beim Eisenbahnbau Lignite zwischen $240 \mathrm{~m}$ und $250 \mathrm{~m} \mathrm{~N}$. N., und damit im Niveau der Schieferkohlen der Grundabfolge 3 angeschnitten worden sind (PILLET 1883: 288, 289, 307; BOURDIER 1961: 44). Diese können dort aber nicht durch Bruchtektonik in ihre heutige Höhenposition geraten sein, weil östlich davon überhaupt keine Quartärablagerungen, sondern nur kreidezeitliche Kalkmergel anstehen.

Die Hauptschieferkohlenflöze der Grundabfolge 3 werden wie die beiden höheren Sedimentationssequenzen von tonigen bis sandigen Feinakkumulationen unterlagert, die hier mindestens $25 \mathrm{~m}$ mächtig sind (Abb. 2). Ihr Liegendes bilden stark verwitterte, nahezu kalkfreie Moränenformationen. Über die Lignite breiten sich wie bei der Grundabfolge 2 diskordant Schotter. Sie werden im Bereich der Banquette von Sonnaz bisweilen von recht breiten und mit Moränenmaterial gefüllten, andererseits aber auch sehr schmalen und nur sandig-toniges Material enthaltenen Eiskeilen durchzogen (HANNSS 1982: 71). Horizontweise enthalten die Schotter zudem aus lokalem 
Kalkschutt bestehendes Murmaterial. Dies verweist sicher auf zeitenweise während ihrer Ablagerung herrschende stadialzeitliche Sedimentationsbedingungen (Abb. 2).

Für alle Grundabfolgen der Banquette im Val du Bourget gilt, daß im Niveau der Schieferkohlen bzw. minerogenen Feinablagerungen auch Schotter anstehen können (HANNSS 1982: 68). Zu erklären wäre dies einmal durch Staffelbrüche bzw. durch Einschachtelung jüngerer in ältere Ablagerungen. Letzteres kann sich beispielsweise im Gefolge eines Gletscherdurchgangs ereignet haben. Es muß jedoch auch damit gerechnet werden, daß im Zuge der Aufsedimentation der Banquette-Ablagerungen entlang der Hauptentwässerungsstränge Schotter akkumuliert wurden, während in größerer Entfernung von ihnen Feinakkumulation vorherrschten, und es dort fallweise auch zur Ausbildung von Mooren gekommen ist. Derartige Prozesse haben sich spätestens seit dem Holozän im Bereich der Leysse-Niederung abgespielt (Abb. 2).

Alles in allem bieten die in den Banquetten des Val du Bourget vorkommenden Schotter hinsichtlich ihrer lithostratigraphischen Klassifizierung die größten Probleme, sieht man von den Grobablagerungen der Grundfolge $1 \mathrm{ab}$, die zumindestens in Pessey-Sonnaz ohne Erosionsdiskordanz die liegenden Feinakkumulationen überdecken.

\section{Die biostratigraphischen Charakteristika der Banquetteablagerungen im Val du Bourget}

\subsection{Die stratigraphische Grundabfolge 1}

Biostratigraphisch zeichnet sich die Grundabfolge 1 dadurch aus, daß über den liegenden Tonen in dem Hauptlignitflöz zwischen $312 \mathrm{~m}$ und $316 \mathrm{~m}$ palynologisch eine Warmphase ( = La Croix-Rouge I-Interstadial) nachgewiesen werden konnte (Tabelle 1). Während dieser Periode waren hinsichtlich der Pollenflora neben den dominierenden Quercus und Ulmus noch Corylus, Fraxinus, Acer, Tilia, Carpinus und Picea stärker vertreten. Im Gegensatz zu den thermophilen Laubwäldern des La Croix-Rouge-I-Interstadials zeichnen sich die vier pollenanalytisch darüber nachgewiesenen Interstadiale, das La Croix-Rouge I- und II- sowie das Sonnaz I- und II-Interstadial, palynologisch durch die Dominanz von Pinus und Picea aus.

Die diese fünf Interstadiale trennenden Stadiale, das Bourget II- bis IV- bzw. Tillet I- und II-Stadial, sind durch einen signifikanten Anstieg der ArtemisiaWerte, z.T. aber auch der Betula-Anteile charakterisiert (GREMmEN,1982, Pollendiagramme 6-8). In den geringmächtigen Sanden und Schottern über den Feinakkumulationen der Grundabfolge 1 sind bisher noch keine Fossilienfunde bekannt geworden.

\subsection{Die stratigraphische Grundabfolge 2}

Die liegenden Feinsedimente des Hauptlignitflözes der stratigraphischen Grundabfolge 2 haben bisher nur wenig biostratigraphische Erkenntnisse gebracht. Eine Ausnahme davon machen die Tone unter den 280 m-Ligniten der Kiesgrube von Voglans (HanNSS 1982: 68). Die von GREMMEN (1982: Pollendiagramm $3,4)$ für diese Tone durchgeführte Pollenanalyse ergab eine eindeutig stadiale Vegetation (= MoulinsStadial) mit $49 \%$ bzw. $64 \%$ Artemisia.

Die Hauptschieferkohlenflöze der stratigraphischen Grundabfolge 2 entstanden nach den von GREMMEN (1982: Pollendiagramme $1-4$ sowie ein noch unveröffentlichtes neues Pollendiagramm von Servolex) durchgeführten palynologischen Untersuchungen während zweier warmzeitlicher Phasen. Damals waren thermophile Laubbäume stark verbreitet. Bei den Warmzeiten handelt es sich von unten nach oben um das $R^{a u}$ des Combes-Interglazial mit den thermophilen Laubgehölzen Carpinus und Corylus bzw. das Tremblay-Interstadial, dessen thermophile Laubgehölze phasenweise stärker von Corylus, Tilia, Ulmus und Quercus geprägt gewesen sind.

Das Bourget I-Stadial, welches das $\mathrm{R}^{\mathrm{au}}$ des CombesInterglazial von dem darüber entwickelten Le Tremblay-Interstadial trennt, ist hinsichtlich seiner Pollenflora durch Pinus, Picea, Betula und Artemisia gekennzeichnet. Das Bourget II-Stadial, das über dem Le Tremblay-Interstadial ausgebildet ist, charakterisiert palynologisch Pinus, Betula und Artemisia.

Mehrfach wurden in den Schottern über den Hauptlignitflözen der stratigraphischen Grundabfolge 2 Makrofossilien geborgen. In der Kiesgrube östlich Le Fort (Banquette von Le Tremblay) wurde in $310 \mathrm{~m}$ N.N. die erste Phalange eines großen Rothirsches (Cervus elaphus; Bestimmung durch H. P. UerP. MANN) gefunden und $6 \mathrm{~m}$ darunter noch Hölzer, die jedoch nicht erhalten geblieben sind.

Auch in der Kiesgrube auf der linken Seite des $\mathrm{R}^{\mathrm{au}}$ des Combes (HanNSS 1982: 94) wurden in den Schottern über den Schieferkohlen in $310 \mathrm{~m}$ Höhe Holzreste von Picea oder Picea/Larix geborgen. Unbestimmbare fossile Hölzer sind in $295 \mathrm{~m}$ Höhe zudem in der Kiesgrube unterhalb Les Ciseaux entdeckt worden, die ebenfalls im Bereich der Banquette von Le Tremblay liegt (HANNSS 1982: 94, 95).

4.2.1 Die Ergebnisse der palynologischen Untersuchung von zwei Schieferkohlenhorizonten der Grundabfolge 2 in Voglans und oberhalb von Le Fromaget

Eine weitere Aufhellung der biostratigraphischen Situation der stratigraphischen Grundabfolge 2 hat 
die pollenanalytische Bearbeitung von drei Schieferkohlenformationen im südlichen Teil der Kiesgrube von Voglans bzw. oberhalb von Le Fromaget gebracht. Die Proben wurden von $\mathrm{CH}$. HANNSS im Gelände geborgen und vom P. PESCHKE pollenanalytisch bearbeitet.

\subsubsection{Methode}

Jede Sedimentprobe war einzeln in PVC-Beuteln verpackt. Schieferkohlen und Lignittone waren noch als kompakte Stücke in den Beuteln, grobkörnige minerogene Sedimente lagen durchweg als Lockermaterial vor. Im Labor wurde von den kompakten Probenstükken der verunreinigte Außenrand entfernt und aus dem Zentrum ein $1-2 \mathrm{~cm}^{3}$ großer Sedimentbestandteil für die Aufbereitung entnommen. Bei Lockermaterial-Proben wurde nach Möglichkeit ein größeres zusammenhängendes Bröckchen für die Aufbereitung ausgewählt. Einige dieser Proben waren aber bereits so stark zerfallen, daß nur noch $1-2$ Teelöffel voll Lockersediment aufbereitet werden konnten. Die Aufbereitung erfolgte nach der im Botanischen Institut der Universität Hohenheim üblichen Methode (HF, modifizierte Schweretrennung, Acetolyse). Der Aufbereitungsrückstand wurde in Glycerin aufgefangen und auch in diesem Medium unter dem Mikroskop analysiert und ausgezählt. Nach Möglichkeit wurde eine Auszählsumme von 700-800 Gesamtpollen (ohne Sporen) angestrebt, was allerdings bei Rückständen aus minerogenen Proben nicht immer möglich war.

Die Ergebnisse der Analyse sind in Gesamtdiagrammen nach gleichem Aufbau dargestellt. Die Gesamtsumme setzt sich aus Baumpollen und Nichtbaumpollen (einschließlich der Cyperaceae) zusammen und bildet die Berechnungsgrundlage (=100\%). Pollen von Wasserpflanzen und Sporen sind wie üblich auf die Gesamtsumme relativ bezogen und in den Diagrammen mit nichtgeschwärzten Kurven dargestellt. Baumpollen mit höheren Werten sind im Gesamtdiagramm mit den gebräuchlichen Symbolen als Zeichenkurven dargestellt, die Werte der übrigen Gehölze sowie die der Kräuter erscheinen in Schattenrissen, teilweise in anderem Maßstab.

Auf den Diagrammen (Abb. 5, 6, 8) und im Text werden folgende Abkürzungen verwendet:

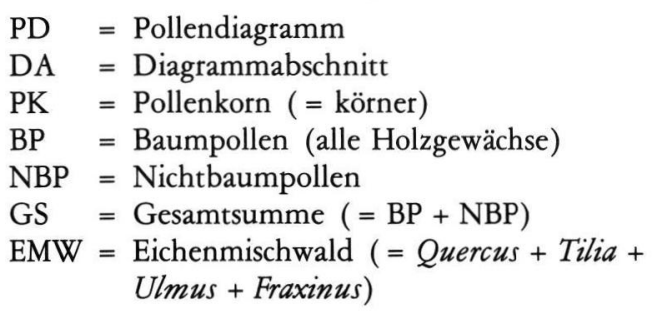

Die Diagrammbeschreibung der einzelnen Profile erfolgt immer von unten nach oben, der Sedimentation entsprechend.

\subsubsection{Die Pollendiagramme}

4.2.1.2.1 $305 \mathrm{~m}$-Lignite oberhalb von Le Fromaget. Proben Nr. 1458-1503

Das beprobte Profil der 305 m-Lignite von Le Fromaget war insgesamt $640 \mathrm{~cm}$ lang und bestand aus Wechsellagen von Ligniten und Tonen (Abb. 4, 5). Die Proben wurden im $5 \mathrm{~cm}$-Abstand der Aufschlußwand entnommen, ihre Pollenführung war sehr unterschiedlich. Der basale Teil des Profils (ab $180 \mathrm{~cm}$ ) war vollkommen pollenfrei, von wenigen zerrissenen Pinus-PK und perisporlosen Farnsporen abgesehen. In dem oberen Tonhorizont erwies sich der Bereich zwischen $35 \mathrm{~cm}$ und $60 \mathrm{~cm}$ ebenfalls als pollenfrei (Abb. 5).

Das Gesamtdiagramm weist im pollenführenden Bereich des Profiles hohe BP-Werte um $80 \%-85 \%$ im Durchschnitt auf, wobei Pinus und Picea am häufigsten vorkommen. Alnus und Betula sind deutlich weniger vertreten. Abies spielt im basalen DA eine gewisse Rolle, EMW-Gehölze und Corylus dagegen in den beiden oberen. Das Diagramm läßt sich in folgende Abschnitte untergliedern:

DA c, $133 \mathrm{~cm}-175 \mathrm{~cm}$ :

Im basalen DA haben Picea und Pinus abwechselnd etwa gleich hohe Werte und stellen den größten Teil des BP. Picea erreicht hier einen Spitzenwert von $46,8 \%$, Pinus von 44,7\%. Betula und Alnus kommen mit deutlich niedrigeren Werten vor, nur nahe der Profilbasis erreicht Alnus einmal einen Wert von $26,8 \%$. Der EMW ist nur mit wenigen Einzelwerten vertreten, welche wohl Fernflug oder Umlagerung zugerechnet werden müssen. Corylus kommt mit geschlossener Kurve vor, ihre Werte liegen aber meist weit unter $1 \%$, so daß der Strauch im Vegetationsbild keine große Rolle gespielt haben kann. Anders verhält es sich mit Abies, welche im gesamten DA mit Werten über $5 \%$ an der GS beteiligt ist (Maximum $11,6 \%$ bei $160 \mathrm{~cm})$. Der NBP ist wenig vertreten, auch Poaceae und Cyperaceae sind nur mit niedrigen Werten daran beteiligt. PK der Korbblütler und deformierte unbestimmbare Pollen sind häufig, was selektive Pollenzersetzung vermuten läßt.

Insgesamt gesehen, weist die Pollenflora des DA c einen mehr oder weniger reinen Nadelwald mit Picea und Pinus auf, in welchem auch Abies vorkam. Die 
(W)

(E)

340
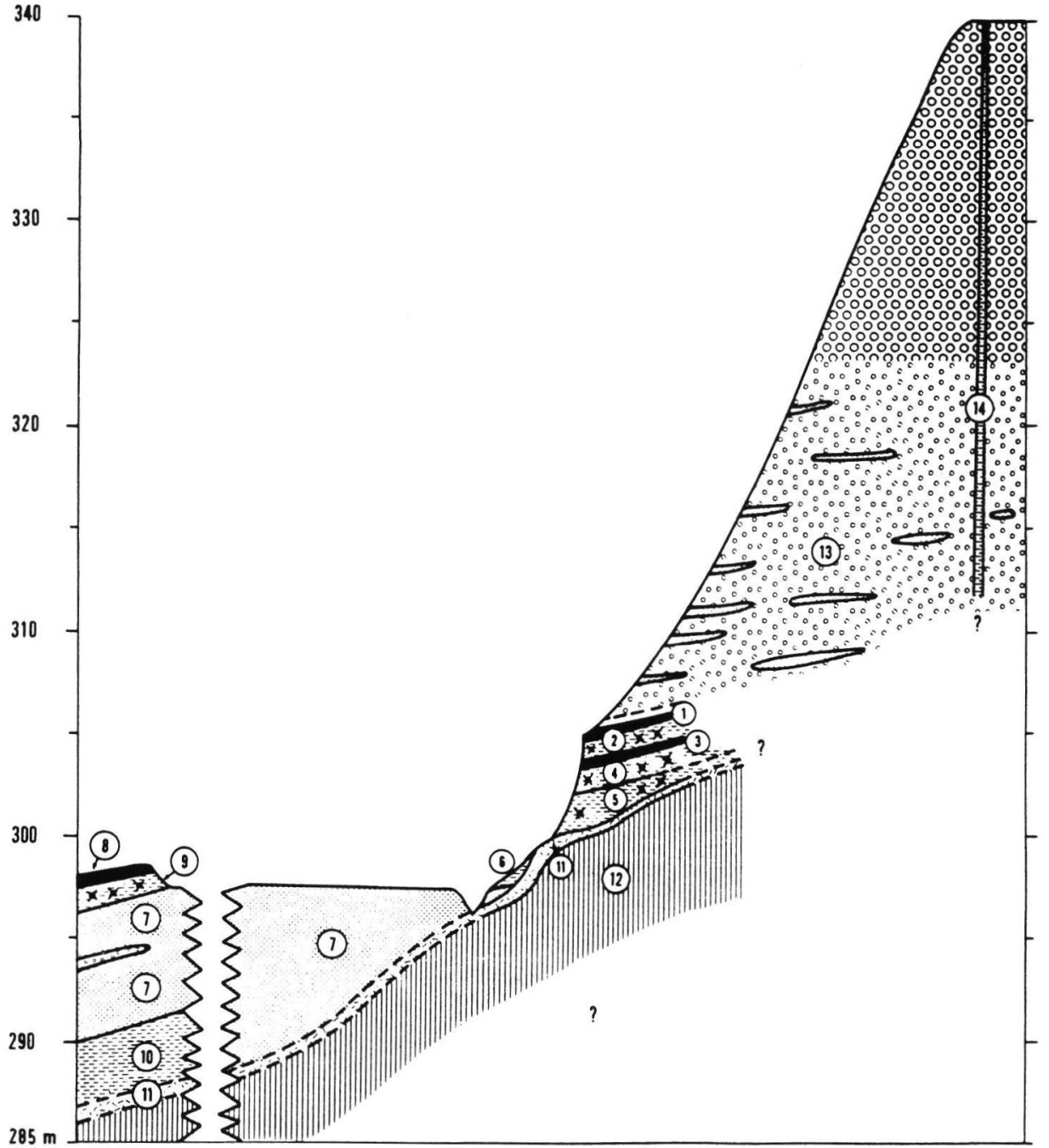

330

Abb. 4: Die Lithostratigraphie der späteemzeitlichen Schieferkohlenformationen und ihrer Decksedimente oberhalb von Le Fromaget (Grundabfolge 2)

1 = Lignite, die sowohl nach unten wie in südlicher Richtung toniger und geringmächtiger werden. Sie enthalten kleine, kantige Geschiebesplitter.

$2=$ Graublaue, ungeschichtete, mitunter leicht siltige Tone. In ihnen stecken Holzreste und kleine, kantige Geschiebesplitter.

3 = Lignite ähnlich der Schicht 1.

4 = Leicht grünliche Tone. Sie sind horizontweise siltig, enthalten kleine, kantige Geschiebesplitter und nach unten spärlicher werdende organische Reste.

5 = Bläuliche Tone mit vereinzelten verkohlten Astresten.

6 = Siltige, orangefarbene Tone.

7 = Graue Mittel- bis Grobsande.

8 = Nach oben hin toniger werdende Lignite, die wahrscheinlich mit der Schicht 3 zu korrelieren sind.

9 = Hellgraue, lagenweise siltige Tone, die mit organischen Resten versetzt sind.

$10=$ Graublaue, plastische Tone.

11 = Verhärtete, stark verwitterte und praktisch kalkfreie Moräne.

12 = Konglomerate des Torton (Miozän).

13 = Nach oben gröber werdende, aus Kalk- und Kristallingeröllen bestehende Schotter.

$14=20 \mathrm{~cm}$ bis $30 \mathrm{~cm}$ breite, mit gelblichen, siltigen Tonen gefüllte Eiskeile. 


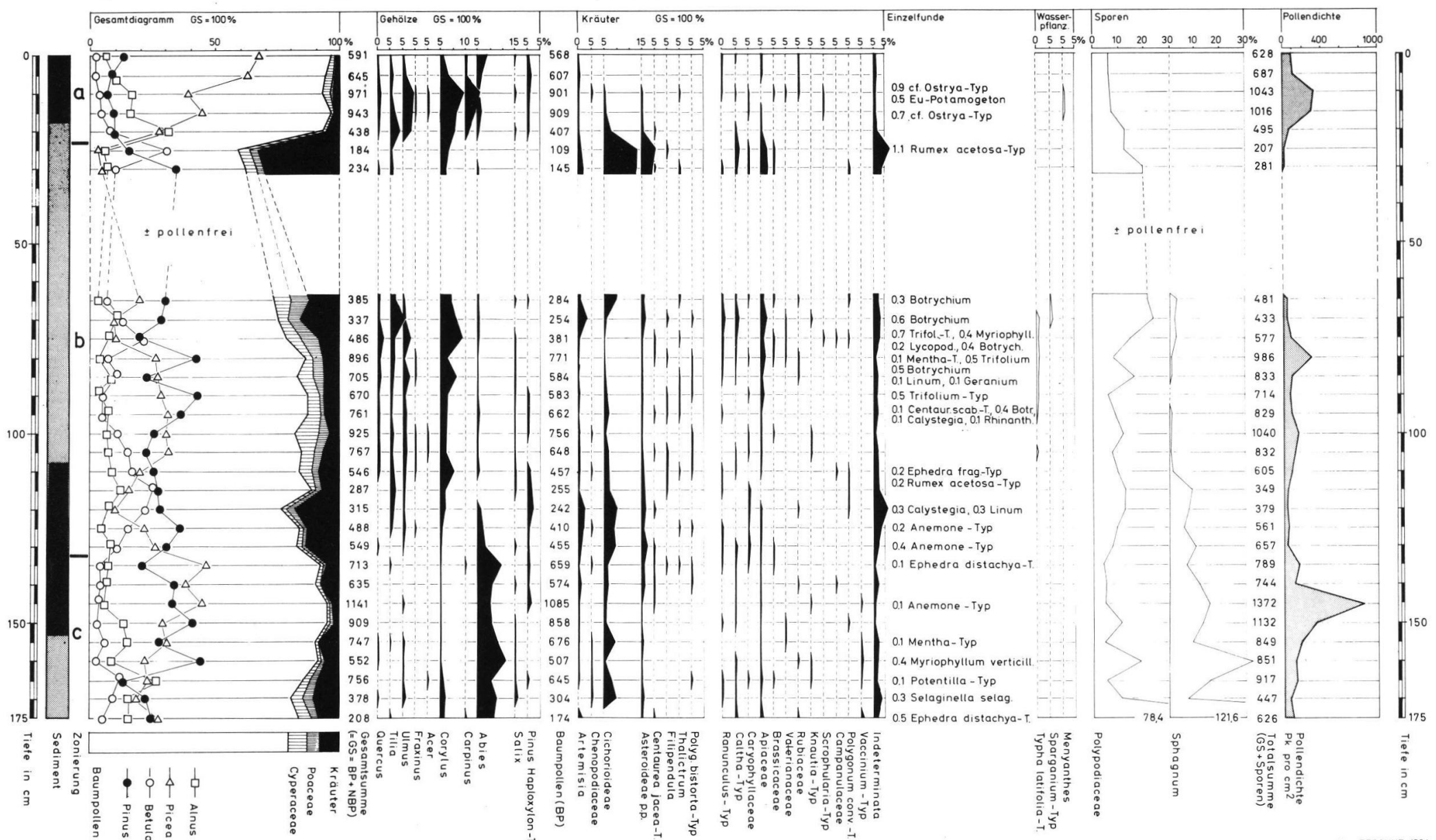


wenigen Laubhölzer, vor allem Alnus, werden wohl nur flußbegleitend bzw. auf Naßstandorten vorgekommen sein.

$\mathrm{DA} \mathrm{b}, 22 \mathrm{~cm}-133 \mathrm{~cm}$ :

Die Pollenflora dieses DA stammt zum größten Teil aus graublauen Tonen, welche horizontweise sandig und mit Geschieben durchsetzt waren. Erst im mittleren Teil der Profilsäule bei $108 \mathrm{~cm}$ erfolgt der Übergang in tonige Lignite mit Feinsandlagen. Die Pollenführung ist hier naturgemäß etwas geringer als im DAc, zwischen $30 \mathrm{~cm}$ und $60 \mathrm{~cm}$ setzt sie sogar ganz aus. Die Pollenerhaltung ist in diesem Sediment ebenfalls schlechter (siehe Anteil der Indeterminata!) und dickwandige PK der Korbblütler sind überrepräsentiert. Dies wirkt sich in einer höheren Beteiligung des NBP am Gesamtdiagramm aus. Bei dem BP hat Picea etwas abgenommen und wird von Pinus meistens überflügelt. Der Anteil von Alnus bleibt unverändert bei Werten um $7 \%-10 \%$. Die Kurve von Betula ist etwas bewegter und weist mit $31,0 \%$ und 25,8\% zwei deutliche Gipfel auf. Vertreter des EMW kommen jetzt mit geschlossenen Kurven vor, auch Corylus ist zahlreicher vorhanden. Abies dagegen hat stark abgenommen, ihr Anteil am Gesamtpollen bewegt sich um $1 \%$.

Im NBP sind jetzt Poaceae und Cyperaceae etwas häufiger, ebenso die Kräuter, wobei auch deren Typenzahl leicht ansteigt.

Das hier erkennbare Waldbild hat sich gegenüber dem DAc etwas gewandelt, Picea hat an Bedeutung eingebüßt und $A$ bies scheint sehr selten geworden zu sein. Dafür kommen jetzt EMW-Gehölze und Corylus zur Ausbreitung und dürften ihnen zusagende Standorte besiedelt haben. Nach dem NBP-Anteil müssen auch offene Flächen in der Landschaft vorhanden gewesen sein, wohl überwiegend Naßstandorte bzw. Versumpfungsflächen, was aus dem Auftreten des Typha-Pollens geschlossen werden kann.

DA a, $0 \mathrm{~cm}-22 \mathrm{~cm}$ :

Im obersten DA ist Picea die dominante Holzart, ihr Pollen erreicht hier mit $67,3 \%$ den Spitzenwert im Profil. Pinus spielt jetzt keine große Rolle mehr, ihre Werte sind auf Anteile um $10 \%$ abgesunken. Alnus und EMW-Gehölze sowie Corylus sind gut vertreten und erstmals kommt auch Carpinus vor. Ihre Pollenkurve ist im gesamten DA geschlossen und weist einen Spitzenwert von $6,2 \%$ auf. Abies ist jetzt wieder häufiger als im DA b, bleibt aber unter $5 \%$.

Der NBP ist artenarm, auch Poaceae und Cyperaceae haben keine große Bedeutung. Der Krautpollen setzt sich nur aus wenigen Typen zusammen.
Somit weist die Pollenflora des DA a auf ein von Picea beherrschtes Waldbild, dem wenig Abies, EMW-Vertreter und Carpinus je nach den edaphischen Möglichkeiten beigesellt waren.

\subsection{8 m-Lignite oberhalb von Le Fromaget. Proben Nr. Fm 1-11}

Das Profil der 298 m-Lignite von oberhalb Le Fromaget war insgesamt $230 \mathrm{~cm}$ lang (Abb. 6). Die obersten $45 \mathrm{~cm}$ bestanden aus tonigen, holzreichen Ligniten, welche hellgrau-bläulichen Tonen mit Schluffresten auflagen. Nur die obersten $15 \mathrm{~cm}$ der Tonablagerungen waren pollenführend, ab $70 \mathrm{~cm}$ bis zur Basis war der Aufbereitungsrückstand so gut wie pollenfrei. Die vereinzelten Pinus-Bruchstücke, Farnsporen und wenig deformierte PK ließen keine Auswertung zu. Die Pollenflora der Tone unterscheidet sich etwas von derjenigen der Lignite, so daß das kurze Diagramm in zwei Abschnitte untergliedert werden kann.

DA b, $45 \mathrm{~cm}-60 \mathrm{~cm}$ :

In den beiden Pollenspektren des DA dominieren im BP Alnus und Picea, nächstwichtige Holzart ist Abies. Neben wenig Pinus und Betula kommen auch die EMW-Gehölze Quercus, Tilia und Ulmus vor, außerdem sind Carpinus, Corylus und Salix mit geringen Werten vertreten.

Der NBP bleibt unter $4 \%$ und kann fast vernachlässigt werden. Die wenigen Kräuter-PK und Gräser lassen keine Aussage zu.

Die hier gefundene Pollenflora läßt einen Fichtenbruchwald mit Erle vermuten. Wenig Edellaubhölzer und Tanne werden höher gelegene Standorte an den Talflanken besiedelt haben.

$\mathrm{DAa}, 0 \mathrm{~cm}-45 \mathrm{~cm}$ :

In den Ligniten läßt der Anteil von Alnus stark nach, ihre Pollenkurve hat nur noch Werte unter $10 \%$. Picea erreicht an der Basis des DA einen Spitzenwert von $66,4 \%$ und geht nach oben hin kontinuierlich auf $23,0 \%$ zurück. Mit Werten um $40 \%$ ist Pinus gut vertreten. Der Abies-Anteil bleibt unverändert, übersteigt aber nicht die 10\%-Marke. EMW-Gehölze, Corylus und Carpinus verschwinden wieder und kommen erst im oberen Bereich mit Werten um 2-3\% zur Geltung. Der NBP hat wegen häufigeren Vorkommens der Cyperaceae etwas zugenommen, auch Kräuter sind jetzt etwas zahlreicher als im DAb. Ihre Artenzusammensetzung ist nichtssagend, der Anteil des unbestimmbaren Pollens liegt oft über $1 \%$. 


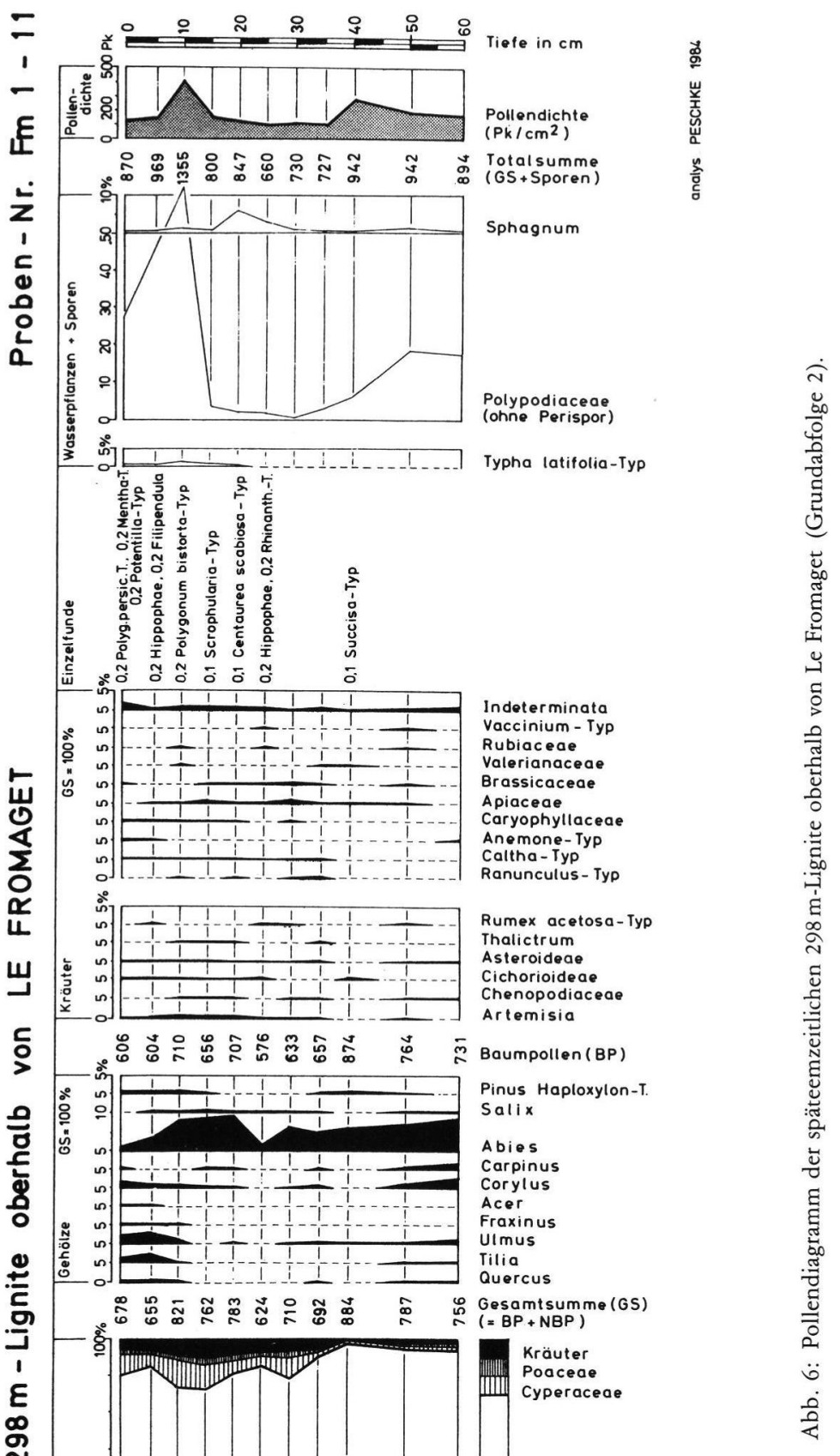




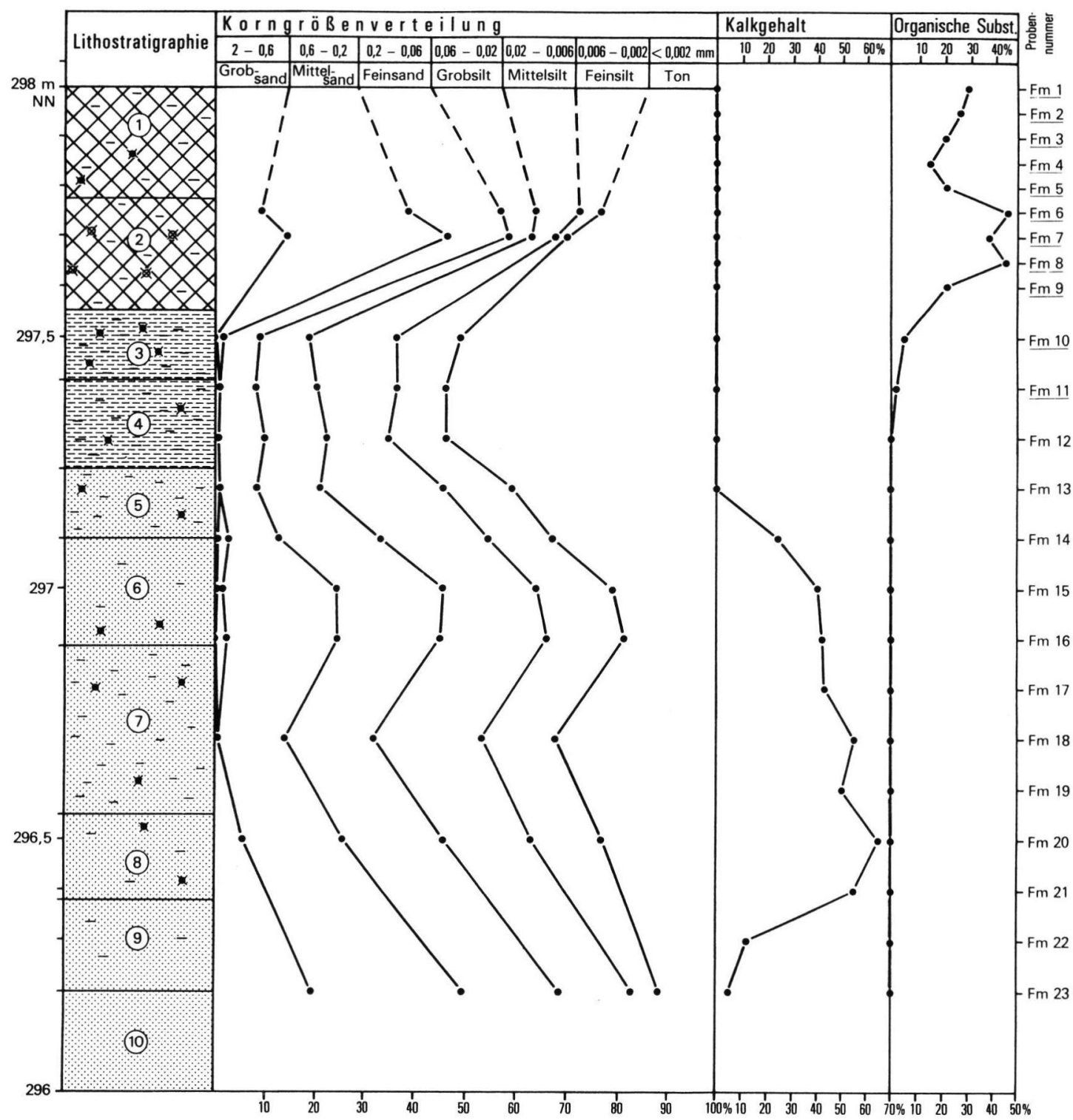

Abb. 7: Lithostratigraphie und Sedimentologie der späteemzeitlichen $298 \mathrm{~m}$-Lignite oberhalb von Le Fromaget (Grundabfolge 2)

Die unterstrichenen Probennummern wurden pollenanalytisch bearbeitet. Siehe auch Abb. 6 .

$1=$ Tonige, holzarme Schieferkohlen.

2 = Tonige, holzreichere Schieferkohlen.

3 = Dunkle Schieferkohlentone mit gepreßten Astresten.

4 = Hellgrau bis leicht bläuliche, stark tonige Feinsedimente mit gepreßten Schilfresten.

$5=$ Hellgrau bis gelbliche, stark tonige Feinstraten mit gepreßten Schilfresten.

6 = Stark siltige, gelb-graue Feinsedimente mit pflanzlichen Makroresten.

7 = Stärker fette, tonige Feinstraten hellgrauer Färbung mit pflanzlichen Makroresten.

8 = Stärker siltige, hellgraue Feinakkumulationen mit pflanzlichen Makroresten.

9 = Stärker sandige Feinsedimente von grünlicher Färbung.

10 = Grau-braune, leicht tonige Mittelsande. 
Die Pollenflora läßt hier einen Fichten-Kiefernwald erkennen, in welchem Tanne, Erle und Birke auf ihnen zusagenden Standorten vorkamen. Vernässungsflächen, auch offenes Wasser, müssen in der Nähe der Lokalität vorhanden gewesen sein. Als Indiz hierfür kann das Vorkommen des Rohrkolben-Pollens (Typha) gelten.

\subsection{6 m-Lignite in der Kiesgrube Voglans, Proben Nr. 1772-1815}

Bei den pollenführenden Schichten der 266 m-Lignite aus dem Süd-Teil der Kiesgrube von Voglans handelt es sich hauptsächlich um tonige Schieferkohlen, deren organischer Anteil bis zu 50\% ausmacht und die zudem sehr kalkarm sind. Nach unten ließ die Pollenführung schlagartig dort nach, wo ab der Probe 1808 die Sandfraktion auf fast $90 \%$ ansteigt (Abb. 3).

Der Aufschluß der $266 \mathrm{~m}$-Lignite in der Kiesgrube Voglans wurde in unterschiedlichen Abständen beprobt, je nach Pollenhöffigkeit der Sedimente (Abb. 8). Drei Proben von der Profilbasis, unter $263,40 \mathrm{~m}$ N.N., welche grünlich-grauen Sanden entnommen wurden, erwiesen sich nach der Aufbereitung als vollkommen pollenfrei. Nicht einmal die sonst üblichen Pinus-Bruchstücke und Farnsporen waren im Rückstand enthalten.

Die Pollenführung der übrigen Proben aus Ligniten und Tonen war zufriedenstellend bis gut. In einigen Proben waren perisporlose Farnsporen in unglaublichen Mengen vorhanden. Im Gegensatz zu den PD aus Le Fromaget ist die Pollenflora hier in Voglans sehr abwechslungsreich und läßt deutlichere Wechsel in der Artenzusammensetzung der Dendroflora erkennen. Das Diagramm wird wie folgt gegliedert:

\section{DAd, $263,45 \mathrm{~m}-263,70 \mathrm{~m} \mathrm{~N}$. N.:}

Hier dokumentiert sich die anspruchsvollste Waldvegetation des gesamten Pollendiagramms: Im BP nimmt Alnus mit über $50 \%$ den Hauptanteil ein (Maximum 60,6\%). Die übrigen Holzarten Picea, Abies und der EMW sind mit etwa gleich hohen Werten zwischen $5 \%$ und $8 \%$ vertreten, Corylus hat an der Basis ein Diagramm-Maximum von 12,8\% . Carpinus ist mit geschlossener Kurve vorhanden, ebenso wie Hedera. Das Vorkommen von Viscum an der Diagrammbasis weist zusätzlich auf entsprechende Klimagunst.

Der NBP liegt bei Werten unter $10 \%, z$.T. mehr als die Hälfte davon entfällt auf Pocaceae und Cypera- ceae. Trotz des hohen Alnus-Anteils am Gesamtpollen sind Zeiger für großflächige Naßstandorte wenig vorhanden, lediglich ein geringes Vorkommen von Sparganium verweist auf offenes Wasser.

Das sich hier abzeichnende Waldbild muß sehr wechselhaft gewesen sein: Die Erle kam häufig als Begleiter der Fließgewässer und im Bruchwald vor. Auf etwas trockeneren Standorten stockte ein Mischwald aus Fichte, Tanne und verschiedenen Edellaubhölzern.

\section{DAc, $263,70 \mathrm{~m}-264,07 \mathrm{~m} \mathrm{~N} . \mathrm{N} .:$}

Hier hat Alnus deutlich an Bedeutung verloren und fällt auf Werte um $10 \% \mathrm{ab}$. Die dominante Holzart in diesem DA ist Picea, welche hier mit 57,6\% ihren Maximalwert des gesamten Profils erreicht. Pinus und Betula sind wie zuvor nur mit geringen Werten um $2 \%-6 \%$ vorhanden. Die EMW-Gehölze sind abgesehen von Ulmus verschwunden. Auch Corylus und Carpinus sind stark zurückgegangen und fehlen in einigen Spektren völlig. Abies kommt dagegen etwas häufiger vor als im DAd und erreicht fast $14 \%$.

Der NBP ist wenig verändert, den größten Anteil hierin haben Cyperaceae. Der Krautpollen ist aussageschwach; deformierte unbestimmbare Pollen sind darin häufig. Von den Wasserpflanzen-PK treten vereinzelt Pollentetraden von Typha auf.

Das sich hier abzeichnende Waldbild ist von der Fichte geprägt, der etwas Tanne zugesellt war. Andere Holzarten sind viel seltener als zuvor. Die Waldvegetation muß jetzt viel eintöniger gewesen sein.

\section{Dab, 264,07 m-264,50m N.N.:}

Der DA b mit Sedimentwechsel der Profilsäule von Ligniten zu grauen Tonen und erneut Ligniten und wieder zu Tonen enthält auch eine andere BP-Zusammensetzung als zuvor: Picea verliert an Bedeutung und geht auf $13,7 \%$ zurück, Pinus ist jetzt der Hauptpollenlieferant. Diese Holzart erreicht hier mit $59,5 \%$ den höchsten Anteil im gesamten Profil. Abies und Alnus gehen weiter zurück und sinken unter $5 \%$. Betula hingegen nimmt zu und erreicht einen Gipfel mit 22,9\%. Im mittleren Bereich des DA sind alle EMW-Arten und Corylus vertreten. In zwei Spektren kommt auch Fagus vor, allerdings nur mit jeweils einem PK.

Der NBP bleibt weiterhin bei niedrigen Werten unter $10 \%$, Cyperaceae sind auch hier am häufigsten vertreten. Der wenige Krautpollen ist etwas artenreicher, aber immer noch aussageschwach. Im gesamten DA 

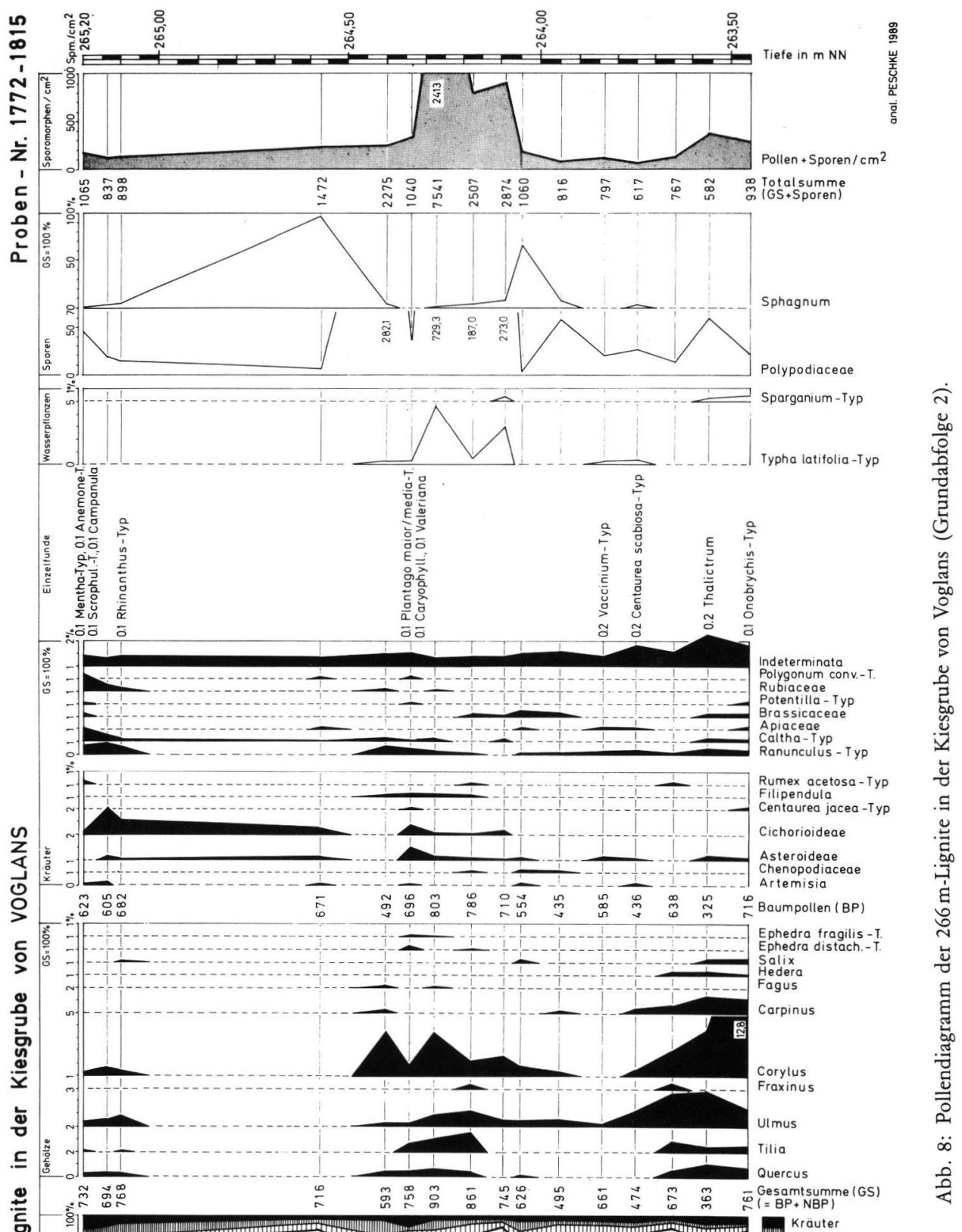

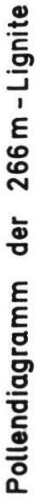

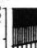

(1)

$=\mathrm{BP}+\mathrm{NBP}$

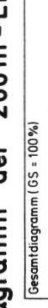

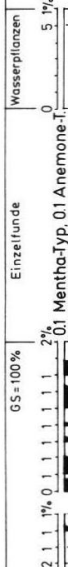


kommen Typha-Tetraden vor, mit einem Spitzenwert von $4,8 \%$. In allen hier analysierten Probenrückständen wurden massenhaft perisporlose Farnsporen gefunden, vereinzelt auch Sporen von Polypodium (im Diagramm nicht verzeichnet). Vermutlich sind ganze Sporangien in das Sediment gelangt, die erst bei der Aufbereitung ihren Sporeninhalt freisetzten.

Das Waldbild hat sich jetzt zu einem Kiefern-Fichtenwald gewandelt, wo auf Sonderstandorten Laubgehölze des EMW und Haselnuß vorgekommen sind. Das Vorhandensein des Pollens von den sehr heliophilen Zwergsträuchern Meerträubel (Ephedra beide Typen) weist darauf hin, daß auch lichte Stellen im Wald vorhanden gewesen sein müssen.

\section{DA a, $264,50 \mathrm{~m}-265,20 \mathrm{~m} \mathrm{~N}$. N.:}

Der oberste DA umfaßt im Profil die längste Strecke, besteht aber nur aus vier Pollenspektren. Die im oberen Teil des Profils anstehenden uneingeregelten Schotter mit taschenförmigen Sandlinsen an ihrer Basis wurden nicht beprobt. Das unterste Pollenspektrum bei $264,58 \mathrm{~m}$ wurde noch aus rötlich-braunen Lignittonen gewonnen, die drei oberen entstammen dunklen Lignittonen.

Im BP dieses Abschnittes gewinnt Picea auf Kosten von Pinus wieder etwas an Bedeutung, auch Abies und Alnus nehmen nach oben wieder zu. Betula ist auf sehr niedrige Werte um $1 \%-2 \%$ zurückgegangen. Andere Laubgehölze fehlen an der Basis vollkommen. Erst in den oberen Lignittonen kommt etwas EMW und Corylus vor.

Im NBP hat sich nicht viel geändert, Cyperaceae und Poaceae stellen immer noch über die Hälfte des Pollenniederschlages. Im Krautpollen dominieren die PK der milchsaftführenden Korbblütler. Der Anteil des unbestimmbaren Pollens schwankt auch hier um $1 \%$. Die hohe Beteiligung der Farnsporen hat nachgelassen. PK von Wasserpflanzen fehlen.

Die Pollenflora des DA a läßt weiterhin einen FichtenKiefernwald mit etwas Tanne vermuten, in welchem zuletzt einige EMW-Gehölze und etwas Erle vorkamen, je nach den Standortbedingungen.

\subsubsection{Die biostratigraphische Einstufung der Pollenprofile von Le Fromaget und Voglans}

Die hier untersuchten Profile weisen alle auf von Nadelhölzern beherrschte Waldzeiten hin, wobei Kiefer und Fichte am häufigsten vorkommen. Da- neben ist aber auch die Tanne in allen Diagrammen mit Maximalwerten um $10 \%-14 \%$ an der Pollenflora beteiligt. Von Erle und Birke abgesehen, sind Laubgehölze oft schwach vertreten und fehlen in etlichen Spektren gänzlich. Somit scheidet eine interglaziale Zeitstellung aller Diagramme - zumindest aus dem Klimaoptimum der Warmzeit - mit Sicherheit aus, denn dann müßten bei der geographischen Lage und Meereshöhe des Val du Bourget thermophile Laubgehölze viel häufiger sein. Die Dominanz der Nadelbäume in den hier vorgelegten Diagrammen weist eher auf interstadiale Klima- und Vegetationsverhältnisse, wie sie am nördlichen Alpenrand in der Schweiz und in Süddeutschland in ähnlichen Sedimenten schon vielfach gefunden worden sind. Die Schieferkohlen bzw. Lignite des schwäbisch-oberbayerischen Alpenvorlandes enthalten meist die Pollenflora borealer Nadelwälder verschiedener FrühwürmInterstadiale (PESCHKE 1983).

Interglaziale Pollenfloren mit typischer thermophiler Laubwaldflora sind eher in limnisch-organogenen Ablagerungen (z. B. Seekreiden) oder Seetonen gefunden worden. Zum biostratigraphischen Vergleich der oft kurzen Pollensequenzen aus den Schieferkohlen fehlt auf der Alpennordseite immer noch ein aus e i n e r Bohrung gewonnenes Vergleichsdiagramm, welches die Vegetationsveränderungen des Jungpleistozäns komplett und lückenlos widerspiegelt. Das lange Profil von Samerberg in Oberbayern (GRÜGER 1979) setzt sich leider aus verschiedenen Bohrungen und Aufschlüssen zusammen und ist nicht frei von Schichtlücken und außerdem in seiner zeitlichen Einstufung noch umstritten. Das Pollenprofil aus der Grande Pile in den Westvogesen (WOILlaRD 1975, 1978) ist wegen der geographischen Lage der Lokalität nur bedingt als Vergleichsdiagramm für oberbayerische Schieferkohlen geeignet.

Für die Pollendiagramme aus dem Val du Bourget sind Vergleichsprofile nördlich der Alpen aus geographischen Gründen ungeeignet, da die heutigen Klimaregionen und Wuchsbezirke zu unterschiedlich sind und sicherlich auch in der Vergangenheit waren. Es muß deshalb auf palynologische Untersuchungen zurückgegriffen werden, welche in den französischen Nordalpen und besonders im Val du Bourget in großer Zahl von GREMMEN (1982) durchgeführt worden sind.

Die Pollensequenzen der hier bearbeiteten Sedimente von oberhalb Le Fromaget und Voglans-Süd lassen sich in vier verschiedenen Waldbildern zusammenfassen und nach ihrer klimatischen Aussage vom ,kühlsten” zum „,wärmsten” Typ wie folgt gliedern: 
Typ I: $\quad$ Fichten-Kiefernwald mit Tanne (um $2 \%$ ) und wenig thermophilen Laubgehölzen:

Le Fromaget 305 m-Lignite DA b Voglans 266 m-Lignite DA a, b.

Typ II: $\quad$ Fichten-Kiefernwald mit Tanne (um 10\%) mit wenig thermophilen Holzarten:

Typ III: $\quad$ Fichten-Tannenwald mit Erle und wenig Thermophilen, sowie sehr wenig Kiefer:

Le Fromaget 305 m-Lignite DA c Le Fromaget $298 \mathrm{~m}$-Lignite DA a.

Voglans 266 m-Lignite DA c.

Typ IV: $\quad$ Fichten-Tannenwald mit Erle, EMW, Haselnuß und Hainbuche; wenig Kiefer:

Le Fromaget 305 m-Lignite DA a. Le Fromaget 298 m-Lignite DA b. Voglans 266m-Lignite DA d.

Diese Waldgesellschaften erlauben im Vergleich mit dem Gesamtdiagramm 7 aus dem Val du Bourget (Fig. 12 in Gremmen 1982) nach der Pollenvergesell-

schaftung - unberücksichtigt der Höhenlage der Lokalität oder petrographischer Belange - folgende Zuordnung:

Typ I: $\quad$ Sonnaz I - Interstadial (PD Sonnaz-Pessey Spektrum Nr. 2-4)

Voglans - Interstadial (PD Voglans I Spektrum Nr. 18-24)

Typ II: $\quad$ Ruisseau des Combes - Interglazial

(PD Ruisseau des Combes Spektrum Nr. 33-36)

Typ III: $\quad$ Tremblay - Interstadial

(PD La Motte-Servolex Spektrum NR. 19-22)

Typ IV: Ruisseau des Combes - Interglazial

(PD Ruisseau des Combes Spektrum Nr. 18-23)

(Zur Chronostratigraphie der Val du Bourget-Interstadiale siehe Tabelle 1 und 2)

Vor wenigen Jahren wurde in einem Moor bei Les Echets in der Nähe von Lyon, ca. $80 \mathrm{~km}$ westlich des Val du Bourget in $267 \mathrm{~m}$ Meereshöhe, ein $39 \mathrm{~m}$ langes Profil erbohrt und palynologisch untersucht (DE BEAULIEU u. REILLE 1984 a, b). Das Pollendiagramm ergab eine zusammenhängende Vegetationsabfolge des Jungpleistozäns vom Ende des Riß bis in das ausklingende Würm. Die einzelnen Vegetationsphasen konnten mit der Grande Pile - Biostratigraphie (WOILLARD 1975, 1978) parallelisiert werden und ergaben weitgehend Übereinstimmung mit der dortigen Zonierung.

Eine Parallelisierung der hier beschriebenen Profile von Le Fromaget und Voglans mit dem Diagramm Les Echets ist allerdings schwierig. Vor allem, weil in Les Echets die Nadelbäume Fichte und Tanne mit wesentlich geringeren Werten auftreten als im Val du Bourget - jedenfalls außerhalb des Klimaoptimums im Eem (Pollenzone B). Möglicherweise ist hier die geographische Lage außerhalb der Alpen dafür verantwortlich und das trocken-warme Klima der Rhone-Niederung wird die vermehrte Ausbreitung der Laubgehölze (Eichenmischwald, Hainbuche, Hasel) begünstigt haben. Bei Berücksichtigung dieser Kriterien kommt für eine Parallelisierung der hier vorgelegten Diagramme eigentlich nur das ausklingende
Eem (Pollenzone B bei $34,5 \mathrm{~m}-34,7 \mathrm{~m}$ ) oder der jüngere Teil der Pollenzone D bei $32,1 \mathrm{~m}-32,5 \mathrm{~m}$ (St. Germain Ic) in Frage.

Hierin bestätigt sich erneut die an oberbayerischen Schieferkohlen gemachte Erfahrung, daß kurze Pollensequenzen biostratigraphisch schlecht mit längeren Pollenprofilen in Übereinstimmung gebracht werden können, da sie sich oft an mehrere Stellen der Pollendiagramme einfügen lassen. Eine einigermaßen zuverlässige chronostratigraphische Eingliederung ist daher nur mit Hilfe anderer Datierungsmethoden und unter Berücksichtigung der sedimentologischen Gegebenheiten der Lokalität möglich.

\subsection{Die stratigraphische Grundabfolge 3}

Wie bereits im Unterkapitel 3.4 beschrieben, ist die Existenz einer dritten, untersten stratigraphischen Grundabfolge 3 lithologisch heute als nahezu gesichert anzusehen. Nur biostratigraphisch läßt sich ihre Existenz bisher noch nicht eindeutig nachweisen.

Dies liegt einmal daran, daß die Pollenanalysen von den Schieferkohlen der Grundabfolge 2 und 3 in der Kiesgrube von Voglans wohl eine ganze Reihe von 
Gemeinsamkeiten, aber auch von Gegensätzen ergeben haben (GREMMEN, 1982, Diagramme 3, 5). Eine eindeutigere biostratigraphische Beweisführung für die Existenz der dritten stratigraphischen Grundabfolge wird unter Umständen die bereits in Angriff genommene neuerliche pollenanalytische Bearbeitung der $250 \mathrm{~m}$-Lignite in der Kiesgrube Aéroport du Bourget bringen.

Auf Grund der neuen lithostratigraphischen Erkenntnisse in der Kiesgrube von Voglans müßte das Hauptlignitniveau der Grundabfolge 3 bereits in das vorletzte Interglazial, das Mindel-Riß-Interglazial zu stellen sein und nicht in ein Riß-Interstadial, wie es der palynologische Bearbeiter der $250 \mathrm{~m}$-Lignite von Voglans, GREMMEN (1982: 81), vorgeschlagen hat.

Das Fehlen von Pollen in den Sanden, Schluffen und Tonen, die unter den Schieferkohlen der Grundabfolge 3 in Voglans-Bouvard und der Kiesgrube Aéroport du Bourget erbohrt worden sind, verweist wahrscheinlich darauf, daß es sich bei ihnen um stadialzeitliche Bildungen, wahrscheinlich der auslaufenden drittletzten alpinen Hauptvereisung, dem klassischen Mindel handelt. (Abb. 2, Tabelle 1).

Wie bei der Grundabfolge 2 wurden in den Schottern über den Ligniten der Grundabfolge 3 Makrofossilien gefunden. In der Kiesgrube von Voglans war es nach Aussagen eines dort tätigen Arbeiters ein $250 \mathrm{~cm}$ langer, $20 \mathrm{~cm}$ dicker und deutlich gekrümmter Stoßzahn, der dieser Schilderung nach eigentlich von einem stadialzeitlichen Mammut (Mammonteus primigenius) herstammen müßte. In der Kiesgrube Aéroport du Bourget wurde über den $250 \mathrm{~m}$-Ligniten neben eher interglazialzeitlichen Relikten von Tannenhölzern und eines Rothirsches (Cervus elaphus) ein wohl interstadialzeitlicher Kiefernstubben geborgen. Wegen seiner zu geringen Länge konnte jedoch nicht entschieden werden, ob es sich bei dem ebenfalls in dieser Kiesgrube gefundenen Stoßzahn um den eines Mammuts (Mammonteus primigenius) oder eines Waldelephanten (Palaeoloxodon antiquus) handelt (HANNSS et al. 1978: 55).

Die Existenz der dritten Grundabfolge im Val du Bourget bestätigt die von SCHLÜCHTER (1987: 113) geäußerte Vermutung, daß nicht die zweit-, sondern die drittletzte Eiszeit die bedeutendste des mittleren Pleistozäns gewesen ist. Dies steht auch im Einklang mit den von Welten (1982: Diagramm 1a) in Meikrich erzielten Ergebnissen und seiner Interpretation durch SCHLÜCHTER (1987: 112).

\section{Die chronostratigraphischen Ergebnisse}

Im allgemeinen bestätigen die zahlreichen konventionellen wie angereicherten ${ }^{14} \mathrm{C}$-Datierungen die ${ }^{234}$ U-230 Th-Altersbestimmungen (Tabelle 2). Bei den Radiokarbondatierungen, die für die letztinterglazialzeitlichen bzw. frühen Fichten-Kiefern-Interstadiale endliche Alter erbracht haben, ist davon auszugehen, daß bei der Probenpräparierung bestimmte organische Verunreinigungen nicht völlig eliminiert werden konnten.

Die Chambéry-Warmzeit, unter der das $\mathrm{R}^{\text {au }}$ des Combes-Interglazial (= Eem) sowie das Tremblay( = St. Germain I) und das La Croix-Rouge-Interstadial (= St.Germain II) von GrEMMEN (1982: Fig. 14) in Tabelle 1 zusammengefaßt wurde, wird nur durch mehrere ${ }^{234} \mathrm{U}-230 \mathrm{Th}$-Datierungen bezüglich ihres Absolutalters erfaßt. Ihnen zufolge müßte die dreigipflige Chambéry-Warmzeit von rund 120000 B.P. bis $90000 \mathrm{~B}$.P. gedauert haben.

Unvereinbar ist das ${ }^{234} \mathrm{U}-230$ Alter von $85000 \pm 5.000$

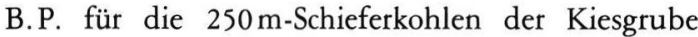
von Voglans mit der bio-, besonders aber lithostratigraphisch gut begründbaren Hypothese, daß es sich dabei um eine präeemzeitliche, möglicherweise bereits Mindel-Riß interglazialzeitliche Warmphase handelt. Chronostratigraphisch konnte also die Existenz der Grundabfolge 3 im Val du Bourget ebensowenig wie eindeutig biostratigraphisch nachgewiesen werden.

\section{Gelöste und ungelöste Probleme der jungpleistozänen Quartärstratigraphie im Val du Bourget}

In den Banquetten des Val du Bourget sind die von oben nach unten älter werdenden Grundabfolgen 1 und 2 entwickelt. Sie überlagern beide transgressiv eine alte, völlig entkalkte Moräne. Die Feinakkumulationen der Grundabfolge 2 gehen dagegen direkt im Liegenden in Glazialablagerungen über, die fast ebenso frisch und kalkreich wirken wie die würmzeitliche Hangendmoräne der Grundabfolge 1.

Die in den beiden oberen Sequenzen enthaltenen Schieferkohlen gehören in der Grundabfolge 2 wahrscheinlich zum Eem und St. Germain I, in der Grundabfolge 1 zum gleichfalls noch einen warmzeitlichen Charakter aufweisenden St. Germain II bzw. vier Fichten-Kiefer-Interstadialen, die wesentlich kühlere Klimaverhältnisse widerspiegeln. Diese Interpretation deckt sich prinzipiell mit den von WOILLARD (1978: 5-8) in der Grande Pile und den von De Beaulieu, ReILLE (1984b: 6-7) in Les Echets erzielten Ergebnissen.

WELTEN (1982, Diagramm 1b) fand unter anderem in Meikirch ebenfalls drei interglazialzeitliche Warmzeiten. Deren oberste stellt er in das Eem, die beiden 
Tabelle 2: ${ }^{14} \mathrm{C}$ - und ${ }^{234} \mathrm{U}$ - und ${ }^{230} \mathrm{Th}$-Datierungen der Schieferkohlenformationen im Val du Bourget. Nach GREMMEN 1982 und HANNSS 1982. Vergleiche auch mit Tabelle 1.

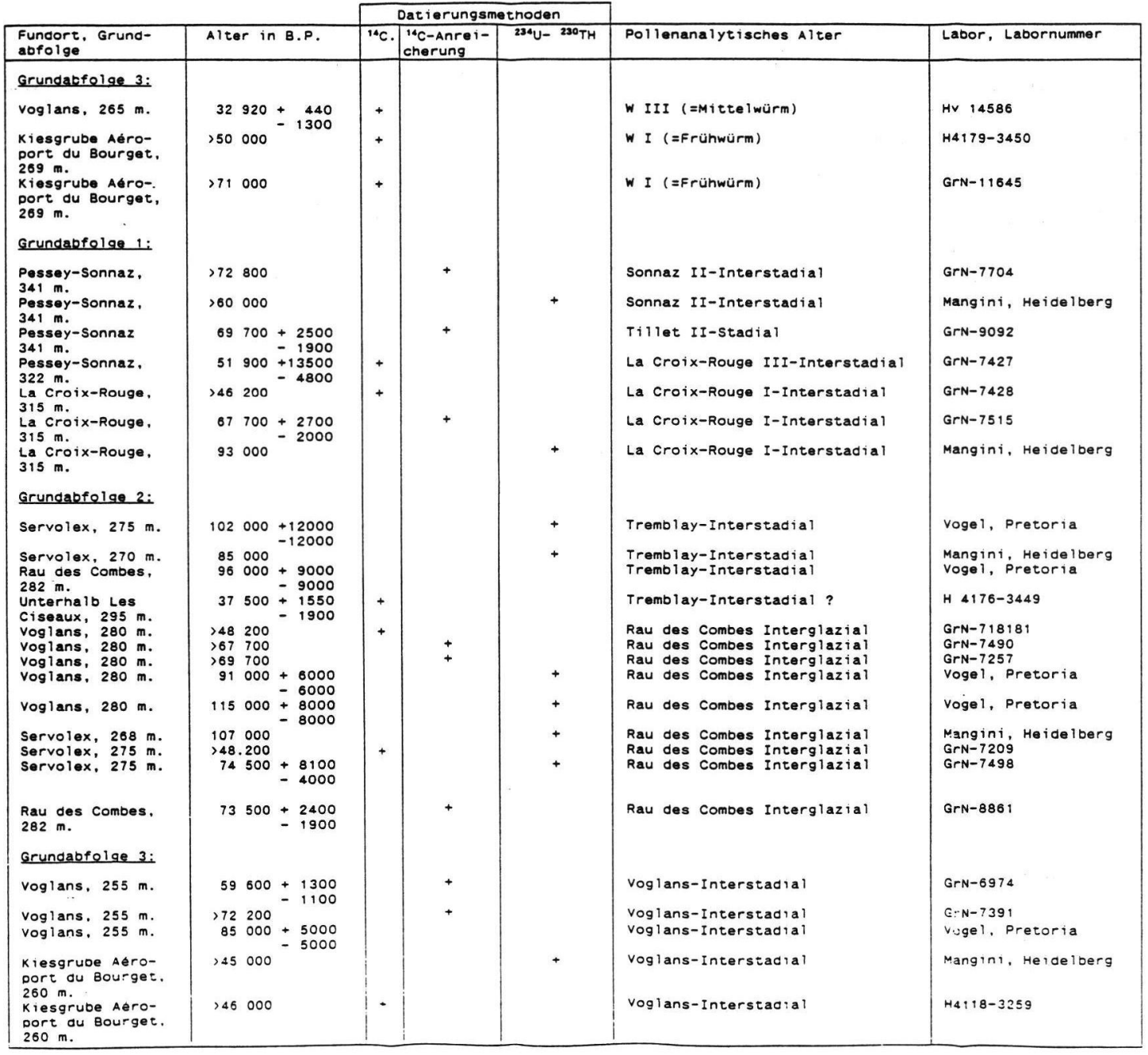

untersten dagegen in das Holstein I und II. Mit dieser Interpretation stimmt im Val du Bourget die Tatsache überein, daß hier bisher nirgends ein einwandfreier stratigraphischer Konnex zwischen der Grundabfolge 2 mit ihren zwei Warmperioden und der hangenden Grundabfolge 1 mit nur einem warmzeitlichen Gipfel beobachtet werden konnte. Nach den im Val du Bourget bisher erzielten chronostratigraphischen Ergebnissen (Tabelle 2) kann die Grundabfolge 2 trotzdem nicht in das Holstein gestellt werden.

Mit ziemlicher Sicherheit ist heute davon auszugehen, daß die $250 \mathrm{~m}-260 \mathrm{~m}$ Lignite im Val du Bourget einer untersten Grundabfolge 3 angehören. Die unter den Schieferkohlen dieser Sequenz anstehende Moräne ist im Gegensatz zu den unter der Grundabfolge 2 und den über der Grundabfolge 1 entwickelten Glazialakkumulationen stark verwittert und nahezu kalkfrei. Sie muß auch wegen dieses sedimentologischen Charakteristikums wesentlich älter als die kalkreiche Liegendmoräne der Grundabfolge 2 und die Hangendmoräne der Grundabfolge 1 sein.

Aus den im Val du Bourget erzielten, vor allem auch lithostratigraphischen Ergebnissen ergibt sich, daß das Ausmaß der glazialen Erosion vor der Akkumulation der Sedimente der Grundabfolge 1 und 2 wesentlich geringer gewesen ist als vor der Sedimentation der die Grundabfolge 3 aufbauenden Akkumulationen. Das läßt sich daraus ableiten, daß die Beckenfüllung des Eisrückzugsees vor der Schieferkohlenbildung im Bereich der Grundabfolge 3 wesentlich mächtiger 
gewesen ist (Abb. 2, Tabelle 1) als die der Grundabfolge 2 soweit sich das in der Kiesgrube von Voglans jüngst beobachten ließ.

Das würde bedeuten, daß nicht das klassische Riß, sondern die Vereisung davor, daß klassische Mindel, in den französischen Nordalpen durch die mächtigeren Gletscher ausgezeichnet war. Zu ganz ähnlichen Ergebnissen ist SCHLÜCHTER (1987: 113) auf der Nordseite der Schweizer Alpen gekommen.

Offene Fragen stellen sich trotz einer relativ großen Anzahl in den letzten Jahren in ihnen gemachter Fossilfunde noch bezüglich der bio- und chronostratigraphischen Position der in den drei Grundabfolgen auftretenden Schotter. Diese gehen nur in der Grundabfolge 1 kontinuierlich über Sande und Schluffe in die liegenden Schieferkohlen über, während in den anderen Grundabfolgen zwischen den Ligniten und hangenden Schottern bis auf zwei Ausnahmen bisher stets mehr oder weniger ausgeprägte fluviatile Erosionsdiskordanzen beobachtet worden sind.

Die im Val du Bourget auftretenden Geröllablagerungen stellen auch deshalb ein stratigraphisches Problem dar, weil sie immer wieder in gleichen absoluten Höhen wie die Feinakkumulationen samt ihren Schieferkohlenformationen auftreten. Möglicherweise sind wenigstens einige der Schotterlagen in die Feinsedimente mit ihren Schieferkohlenniveaus nach Phasen der glazialen Erosion eingeschachtelt worden. Es muß aber auch damit gerechnet werden, daß das Vorkommen von Fein- und Grobablagerungen auf gleicher Höhe auf räumlich ungleiche Sedimentationsprozesse in den spätpleistozänen Hauptentwässerungsläufen zurückgeht. Auf Staffelbrüche dürften hingegen die wenigsten dieser Eigenheiten in Bezug auf die Verbreitung der Schotter in den Banquettes des Val du Bourget zurückzuführen sein.

\section{Schriftenverzeichnis}

Beaulieu, J.-L. DE \& Reille, M. (1984 a): A long Upper Pleistocene pollen record from Les Echets near Lyon, France. - Boreas 13: 111-132, 6 Abb.; Oslo (Universitetsforlaget).

Beaulieu, J.-L. DE \& Reille, M. (1984 b): The pollen sequence of Les Echets (France): a new element for the chronology of the Upper Pleistocene. - Géographie Physique et Quarternaire 38: 3-9, 2 Abb.; Paris (Presses de l'Université de Montréal).

BOURDIER, F. (1961): Le bassin du Rhône au Quaternaire. 364 S., 296 Abb.; Paris (Editions du CNRS).

Debelmas, J. (1970): Alpes (Savoie et Dauphiné). 213 S., 119 Abb.; Paris (Masson \& Cie).

Gremmen, W. H. E. (1982): Palynological investigations on late Pleistocene deposits in southeastern France. 94 S., 14 Abb., 3 Tab., 11 Tafeln; Groningen.
GrÜGER, E. (1979): Spätriß, Riß/Würm und Frühwürm am Samerberg in Oberbayern - ein vegetationsgeschichtlicher Beitrag zur Gliederung des Jungpleistozäns. Geologica Bavarica 80: 5-64, 3 Abb., 6 Tab., 3 Tafeln; München (Bayerisches Geologisches Landesamt).

Hannss, CH. (1982): Spätpleistozäne bis postglaziale Talverschüttungs- und Vergletscherungsphasen im Bereich des Sillon alpin der französischen Nordalpen. - Mitteilungen der Kommission für Quartärforschung der Österreichischen Akademie der Wissenschaften, 4: 213 S., 58 Abb., 18 Tab., 13 Tafeln; Wien (Österreichische Akademie der Wissenschaften).

Hannss, Ch., Koenigswald, von W. \& A. MillionRousseau, A. (1978): Découvertes d'ossements fossiles dans la sablière au SSW de la base du Bourget-du-Lac (Savoie). - Annales du Centre Universitaire de Savoie, Sciences Naturelles, 3: 53-57, 1 Abb.; Chambéry.

Nicoud, G. (1979): Deux exemples de difficultés recontrées par le génie civil dans le Quaternaire chambérien. Documents du BRGM, Colloque National, Lyon 13-14 mars 1979, Connaître le Sous-Sol, un Atout pour l'Aménagement Urbain, 2: 859-872, 9 Abb.; Lyon.

PeschKe, P. (1983): Palynologische Untersuchungen interstadialer Schieferkohlen aus dem schwäbisch-oberbayerischen Alpenvorland. - Geologica Bavarica 84: 69-99, 8 Abb., 1 Tafel; München (Bayerisches Geologisches Landesamt).

Pillet, L. (1883): Etude sur les terrains quaternaires de l'arrondissement de Chambéry. - Mémoires de l'Academie Impériale de Savoie, série 3: 285-337, Chambéry.

Renault-Miskovsky, J. (1986): Relations entre les spectres archéo-polliniques du Sud-Est de la France et les oscillations climatiques entre 125000 ans et le maximum glaciaire. - Bulletin de l'Association Française pour l'Etude du Quaternaire, 25-26: 56-62, 2 Abb.; Paris (Maison de la Géologie).

SCHAEFer, I. (1981): Die glaziale Serie. Gedanken zum Kernstück der alpinen Eiszeitforschung. - Zeitschrift für Geomorphologie N.F., 25: 271-289, 6 Abb.; Berlin (Gebrüder Bornträger).

SCHLÜCHTER, CH. (1987): Talgenese im Quartär, eine Standortbestimmung. - Geographica Helvetica, 42: 109-115, 2 Abb.; Zürich (Fotorotar).

WeLtEN, M. (1982): Pollenanalytische Untersuchungen im Jüngeren Quartär des nördlichen Alpenvorlandes der Schweiz. Diagrammheft. Beiträge zur Geologischen Karte der Schweiz, N. F., 156: 44 Tafeln, Bern.

WOILLARD, G. M. (1975): Recherches palynologiques sur le Pleistocène dans l'Est de la Belgique et dans les Vosges Lorraines. - Acta Geographica Louvaniensia 14: 118 S., 7 Abb., 8 Tab., 40 Tafeln; Louvain-La-Neuve.

- (1978): Grande Pile peat bog: a continous pollen record for the last 140000 years. - Quaternary Research 9: 1-21, 5 Abb., 1 Tab., New York (Academic Press).

— \& MooK, W. (1982): Carbon-14 dates at Grande Pile: Correlation of land and sea chronologies. - Science, 215: 59-161, 2 Abb.; Washington (American Association for the Advancement of Science).

Manuskript eingegangen am 28.03. 1991 